\title{
Infrastrukturen (Energie- und Wasserversorgung)
}

Markus Groth, Julia Rose

9.1 Einleitung - 194

9.2 Energieversorgung - 195

9.2.1 Stand des Wissens zu klimawandelbedingten Betroffenheiten der Energieversorgung und -infrastruktur in Deutschland - 195

9.2.2 Struktur der Energieversorgung und -infrastruktur in Hamburg - 197

9.2.3 Mögliche Auswirkungen des Klimawandels auf die Energieversorgung und -infrastruktur in Hamburg - 198

9.2.4 Zwischenfazit - 199

9.3 Wasserversorgung - 200

9.3.1 Stand des Wissens zu klimawandelbedingten Betroffenheiten der Wasserversorgung und -infrastruktur in Deutschland - 200

9.3.2 Struktur der Wasserversorgung und Wasserversorgungsinfrastruktur in Hamburg - 202

9.3.3 Mögliche Auswirkungen des Klimawandels auf die Wasserversorgung in Hamburg - 203

9.3.4 Zwischenfazit - 204

$9.4 \quad$ Fazit -205

Literatur - 205 


\subsection{Einleitung}

Mit der Energie- und Wasserversorgung werden in diesem Kapitel zwei Sektoren betrachtet, denen jeweils eine große gesamtgesellschaftliche Bedeutung zukommt. Sie werden daher auch als Kritische Infrastrukturen (Bundesministerium des Innern 2009) bezeichnet, denn ihr Ausfall oder ihre Beeinträchtigung kann zu nachhaltig wirkenden Versorgungsengpässen, erheblichen Störungen der öffentlichen Sicherheit oder anderen Problemen führen (Deutscher Bundestag 2011). Die grundsätzliche Relevanz von Auswirkungen klimatischer Veränderungen auf Kritische Infrastrukturen wie beispielweise die Energieversorgung ist zunehmend Teil der wissenschaftlichen Diskussion und auch für Deutschland erkennbar (z. B. Rubbelke und Vogele 2011; Schaeffer et al. 2012; Vine 2012; van Vliet et al. 2012; Groth und Cortekar 2015). Bei der Analyse möglicher Verletzlichkeiten dieser Kritischen Infrastrukturen sind grundsätzlich alle Gefahren relevant, die zu einer Unterbrechung der Versorgung führen können. Aspekte des Klimawandels und damit verbundene Folgen werden vor allem unter dem Oberbegriff der Naturgefahren diskutiert (Birkmann et al. 2010).

Auch wenn mit entsprechenden Einschränkungen oder Ausfällen Kritischer Infrastrukturen ganz unterschiedliche Auswirkungen wie eine Beeinträchtigung der Lebensqualität, Gesundheitsgefahren oder auch Gefahren sozialer Unruhen verbunden sein können (Bundesministerium des Innern 2009), zeigen doch vor allem ökonomische Abschätzungen die entsprechende Bedeutung ganz unmittelbar auf. So werden monetäre Schäden, die durch Versorgungsunterbrechungen der Energieversorgung auftreten können, bereits in einigen Studien eindrucksvoll aufgezeigt. Eine deutschlandweite einstündige Versorgungsunterbrechung an einem Werktag im Winter könnte beispielsweise volkswirtschaftliche Kosten zwischen 0,6 und 1,3 Mrd. $€$ verursachen. Ein ganztägiger Ausfall würde zu volkswirtschaftlichen Kosten von bis zu $30 \mathrm{Mrd}$. € führen (Bothe und Riechmann 2008). Es sind also nicht nur Versorgungsunterbrechungen von langer Dauer, die hohe Schäden bewirken. Bereits kurze Versorgungsunterbrechungen von wenigen Sekunden bis Minuten können beträchtliche Schäden verursachen. Diese treten insbesondere dort ein, wo hoch synchronisierte Produktionsketten (z. B. in der Papierindustrie) betroffen sind. Die durchschnittlichen direkten Kosten für einen einstündigen Stromausfall könnten sich differenziert nach Landkreisen, Städten, Tageszeiten, verschiedenen Wirtschaftszweigen und privaten Haushalten beispielsweise für Berlin auf rund 15 Mio. $€$ und für Hamburg auf 12,5 Mio. $€$ belaufen (Piaszeck et al. 2013). Eine nach Regionen und Sektoren differenzierende Studie kommt zu dem Ergebnis, dass eine stromausfallbedingt fehlende Gigawattstunde zu durchschnittlichen Ausfallkosten von etwa 7,6 Mio. $€$ führt. Die durchschnittlichen Kosten eines einstündigen deutschlandweiten Stromausfalls belaufen sich demnach auf rund 430 Mio. $€$ (Growitsch et al. 2015).

Bei derartigen ökonomischen Bewertungen ist die Ursache einer Versorgungsunterbrechung zunächst nicht relevant. Auch stehen ökonomische Abschätzungen ausschließlich klimawandelbedingter Auswirkungen auf die Energie- und Wasserver- sorgung für Deutschland bisher noch nicht im Fokus wissenschaftlicher Untersuchungen. Dennoch sind entsprechende Rückschlüsse auch auf mögliche Versorgungsunterbrechungen zu ziehen, die durch veränderte Klimabedingungen (mit) verursacht werden.

Das Kapitel ist wie folgt aufgebaut: Der zunächst betrachtete Infrastrukturbereich ist die Energieversorgung. Dabei wird exemplarisch auf die Stromversorgung fokussiert. Die Wärmeund die Gasversorgung werden nicht explizit analysiert, da sie weniger kritisch für das Funktionieren einer Gesellschaft sind als die Stromversorgung und gleichzeitig als weniger anfällig für Folgen des Klimawandels einzuschätzen sind. Da bzgl. der Stromversorgung noch keine Untersuchungen vorliegen, die sich speziell mit der Energieversorgung in Hamburg und der Metropolregion befassen, wird das folgende Vorgehen gewählt: Zunächst wird basierend auf bereits vorliegenden Untersuchungen (Cortekar und Groth 2013, 2015; Groth und Cortekar 2015) ein Überblick über den aktuellen Wissensstand zu physikalischen Betroffenheiten des deutschen Energiesektors entlang zentraler Stufen der Wertschöpfungskette (Gewinnung und Verfügbarkeit, Erzeugung und Umwandlung sowie Verteilung und Netze) sowie für die unterschiedlichen Energieträger (fossile und erneuerbare) gegeben. Danach wird der Fokus auf die Auswirkungen des Klimawandels auf die Energieversorgung in Hamburg gelegt. Im Zuge dessen wird der Stand des Wissens zur klimawandelbedingten Betroffenheit des Energiesektors auf die Struktur der Energieversorgung in Hamburg übertragen. Die zentralen Ergebnisse für diesen ersten Bereich der Versorgungsinfrastrukturen werden in einem Zwischenfazit zusammengefasst, wobei zudem bestehende Wissenslücken und weitere Wissensbedarfe skizziert werden.

Im zweiten Teil des Kapitels wird insbesondere die Wasserversorgung in Hamburg und ergänzend auch die Abwasserentsorgung betrachtet, da diese Bereiche oft eng zusammenhängen und in vielen Studien parallel untersucht werden. Somit umfasst - Abschn. 9.3 die zwei großen Bereiche der Siedlungswasserwirtschaft. Der Aufbau ist ähnlich dem der Energieversorgung. Zunächst wird allgemein auf die Auswirkungen des Klimawandels auf die Wasserversorgung eingegangen. Der Klimawandel kann dabei sowohl Auswirkungen auf den Wasserkreislauf und damit das Angebot von Wasser haben als auch auf die Infrastrukturanlagen und den Verbrauch von Haushalten, Industrie und Gewerbe. In diesem Teil werden vorhandene Studien und Arbeiten aus anderen Regionen Deutschlands sowie teilweise aus dem Ausland herangezogen. Anschließend wird auf die Struktur der Wasserversorgung in Hamburg eingegangen, mit Informationen zur Wassergewinnung und -verteilung sowie zur Abwasserentsorgung. Danach werden die wenigen verfügbaren Untersuchungen für Hamburg und umliegende Regionen vorgestellt, die es zu den Auswirkungen des Klimawandels auf die Wasserversorgung gibt. In einem Zwischenfazit werden die bestehenden Erkenntnisse zusammengeführt. 


\subsection{Energieversorgung}

\subsubsection{Stand des Wissens zu \\ klimawandelbedingten Betroffenheiten \\ der Energieversorgung und -infrastruktur in Deutschland}

\subsubsection{Auswirkungen des Klimawandels auf die Energiegewinnung und -verfügbarkeit}

\section{Konventionelle Energieträger}

Bei der Gewinnung von Öl und Gas muss zunächst grundsätzlich zwischen der On- und Offshore-Förderung unterschieden werden (Groth und Cortekar 2015). Während für die Onshore-Förderung derzeit keine nennenswerten Beeinträchtigungen durch den Klimawandel identifiziert werden können, ist hinsichtlich der Offshore-Förderung der Meeresspiegelanstieg ein mögliches Risiko. Diese Gefährdung bezieht sich vor allem auf die Förderanlagen (Lauwe 2009; Scheele und Oberdörffer 2011; Bardt et al. 2013). Zudem kann es bei der Offshore-Förderung von Öl und Gas zu Herausforderungen durch eine mögliche Zunahme von Extremwetterereignissen kommen (Kuckshinrichs et al. 2008; Hirschl und Dunkelberg 2009).

Der Abbau von Braunkohle (ausschließlich) und Uran (überwiegend) erfolgt in Tagebauen. Um einer potenziellen Beeinträchtigung der Anwohner von Braunkohletagebauen durch Staubentwicklung nach längerer Trockenheit entgegenzuwirken, muss die Braunkohle befeuchtet werden, wobei hierfür ausreichend Wasser zur Verfügung stehen muss (Bardt et al. 2013). Mögliche Auswirkungen auf die Wasserhaltung bei Braunkohletagebauen sind aufgrund von Extremereignissen wie Hitzeperioden oder lang anhaltenden hydrologischen und meteorologischen Dürren zu erwarten (Kuckshinrichs et al. 2008; Hirschl und Dunkelberg 2009). Eine ausreichende Wasserverfügbarkeit ist eine wichtige Herausforderung, da die Braunkohlekraftwerke zumeist über das abgepumpte und sonst in den Tagebau nachsickernde Grundwasser gekühlt werden (Groth und Cortekar 2015). Auch wenn eine lange Trockenheit zu keiner Beeinträchtigung der Förderung führt, so können Starkregenereignisse aufgrund von Erosion und Bodendegradierung zu Abrutschungen im Tagebau führen.

Der Abbau von Steinkohle erfolgt unter Tage. Hier sind derzeit keine klimawandelbedingten Beeinträchtigungen der Gewinnung in Deutschland zu erwarten. Deutschland ist jedoch auch auf Steinkohleimporte angewiesen, und es sind durchaus negative Auswirkungen auf die Förderung sowie den Transport von Steinkohle in und aus anderen Regionen der Welt zu erwarten (Bardt et al. 2013).

Neben der als eher gering eingeschätzten Betroffenheit bei der Gewinnung der Rohstoffe werden klimawandelbedingte Beeinträchtigungen des Transports der Energieträger zu den Kraftwerken am ehesten dort erwartet, wo die zugrunde liegende Infrastruktur sensibel auf klimatische Veränderungen reagiert (Cortekar und Groth 2013). Dies kann beispielsweise die Schieneninfrastruktur sein, wobei in der Literatur in erster Linie die besondere Relevanz des Transports mit Schiffen hervorgehoben wird; so kann die Schiffbarkeit auf Binnenwasserstraßen durch zu hohe oder zu niedrige Pegelstände beeinträchtigt werden (Bundesministerium für Verkehr, Bau und Stadtentwicklung 2007; Bundesregierung 2008; Hirschl und Dunkelberg 2009; Lauwe
2009; Scheele und Oberdörffer 2011; Bardt et al. 2013). Dieser Effekt ist in erster Linie bei der Versorgung von Steinkohlekraftwerken zu sehen (Kuckshinrichs et al. 2008). Ein weiteres grundsätzliches Gefährdungspotenzial für die Rohstoffversorgung besteht insbesondere in einer möglichen Betroffenheit von Pipelines durch Extremwetterereignisse (Hirschl und Dunkelberg 2009).

\section{Regenerative Energieträger}

Für die Nutzung von Biomasse zur Energieerzeugung zeichnen sich zwei durch klimatische Einflüsse bedingte Betroffenheiten ab:

Einerseits könnten sich aufgrund trockener werdender Sommer die Anbauperioden und damit potenziell auch die Verfügbarkeit von Energiepflanzen verschieben (Kuckshinrichs et al. 2008; Scheele und Oberdörffer 2011). Hinzu kommt, dass die Energie gegenwärtig noch zu einem überwiegenden Anteil aus den Pflanzen selbst und nicht aus deren Abfallstoffen gewonnen wird. Hier könnte sich eine zunehmende Konkurrenz zur Nahrungsmittelnutzung abzeichnen (Bardt et al. 2013), die in Lieferengpässen resultieren kann.

Andererseits können Extremwetterereignisse das Angebot an Biomasse beeinträchtigen (Bundesregierung 2008; Scheele und Oberdörffer 2011). Im Zuge dessen könnten beispielsweise auch Lebensmittelkrisen durch den klimawandelbedingten Ausfall von Anbauflächen verschärft werden. Diese Effekte müssen sowohl auf einem globalen als auch regionalen Maßstab betrachtet werden und wirken sich tendenziell negativ aus (Cortekar und Groth 2013).

Der Flusspegel beeinträchtigt nicht nur die Schiffbarkeit, sondern auch die Energieerzeugung in Wasserkraftanlagen, sowohl durch zu viel als auch zu wenig verfügbares Wasser. Unterschieden werden muss hier zwischen Laufwasserkraftwerken und Speicherkraftwerken, wobei Laufwasserkraftwerke sensibler auf zu niedrige oder zu hohe Pegelstände reagieren. Dies führt bestenfalls nur zu einer reduzierten Auslastung, schlimmstenfalls müssen die Anlagen ganz abgeschaltet werden (Kuckshinrichs et al. 2008; Finley und Schuchard 2009; Lauwe 2009; Scheele und Oberdörffer 2011; Bardt et al. 2013).

Hinsichtlich der Sonnenenergie muss zwischen Photovoltaikanlagen und solarthermischen Kraftwerken unterschieden werden (Groth und Cortekar 2015). Während Photovoltaikanlagen beispielsweise auch bei bewölktem Himmel Energie erzeugen, liefern solarthermische Kraftwerke nur bei direkter Sonneneinstrahlung Energie. Für Solaranlagen findet sich in der Literatur die Einschätzung, dass insgesamt mit einer zunehmenden Leistung im Sommer zu rechnen ist (Scheele und Oberdörffer 2011; Bardt et al. 2013). Für die Wintermonate zeigen sich unterschiedliche Einschätzungen. So kommen Bardt et al. (2013) zu dem Ergebnis, dass im Winter mit einer Abnahme der Kapazitäten zu rechnen sei, während Scheele und Oberdörffer (2011) zu der Einschätzung gelangen, dass sich die Voraussetzungen für solarthermische Kraftwerke aufgrund einer Abnahme der Schneemenge, der Schneebedeckung und der Schneetage insbesondere in den Wintermonaten grundsätzlich verbessern könnten. Im Rahmen des europäischen Forschungsprojektes IMPACT2C wurden mögliche Auswirkungen eines globalen Temperaturanstiegs um 2 Grad auf den Energiesektor untersucht, wobei die Schlussfolgerung dort lautet, dass die Erträge von Photovoltaikanlagen im Zuge des Klimawandels eher abnehmen werden (IMPACT2C 2016). 


\subsubsection{Auswirkungen des Klimawandels auf die Energieerzeugung und -umwandlung Konventionelle Energieträger}

Die Energieerzeugung aus konventionellen Energieträgern ist derzeit - und wird zukünftig - insbesondere durch zwei Faktoren beeinträchtigt: zum einen durch die bereits erwähnte Verfügbarkeit und Temperatur von Wasser zur Kühlung der Kraftwerke (Förster und Lilliestam 2010; Linnerud et al. 2011) und zum anderen durch die insbesondere in den Sommermonaten steigenden Durchschnittstemperaturen (sowohl der Luft als auch des Wassers). Beide Faktoren beeinträchtigen allerdings nicht die Energieerzeugung aus einem bestimmten Energieträger, sondern vielmehr die Erzeugung in einem bestimmten Kraftwerkstyp (Buth et al. 2015).

Von den beiden genannten Einflussgrößen sind Braunkohlekraftwerke am geringsten betroffen. Zwar handelt es sich hierbei - wie bei Steinkohle, Erdöl, Erdgas und auch Kernenergie - um Kraftwerke, die auf die Verfügbarkeit von Kühlwasser angewiesen sind, es zeigen sich jedoch unterschiedliche Einschätzungen bzgl. der Betroffenheit (Cortekar und Groth 2013). Während Hirschl und Dunkelberg (2009) die Leistungsfähigkeit aller thermischen Kraftwerke grundsätzlich durch ein im Sommer geringeres Wasserdargebot (sowohl Grund- als auch Flusswasser) zur Kühlung betroffen sehen, kommen Bardt et al. (2013) zu dem Ergebnis, dass Braunkohlekraftwerke zur Kühlung in erster Linie das aus dem meist nahe gelegenen Tagebau abgepumpte Wasser verwenden und damit weniger von der Kühlwasserproblematik betroffen seien. Sie sehen die Leistungsfähigkeit eher durch hohe Temperaturen beeinträchtigt, die physikalisch gering, aber wirtschaftlich spürbar seien. Die Wirkungsgradverluste aufgrund hoher Außentemperaturen sind bei allen thermischen Kraftwerkstypen gleichermaßen zu erkennen, bei Gaskraftwerken aber besonders deutlich (Engelhard 2011; Wachsmuth et al. 2012; Bardt et al. 2013).

Anders als Braunkohlekraftwerke, die sich meist in der Nähe der Tagebaue befinden, stehen andere thermische Kraftwerke (Steinkohle, Gas, Öl, Kernkraft) häufig an Flüssen. Dies sichert zum einen die gute logistische Versorgung mit den Rohstoffen via Schiff, zum anderen ist die Verfügbarkeit von Kühlwasser aus dem Fluss gegeben (Groth und Cortekar 2015). In Hitzeperioden können die Wasserentnahmemengen aufgrund sinkender Flusspegel allerdings eingeschränkt sein (Finley und Schuchard 2009). Zudem darf das wieder eingeleitete Wasser aus wasserrechtlichen Gründen eine bestimmte Temperatur nicht überschreiten, die im Sommer in der Regel ohnehin schon erhöht ist (Groth und Cortekar 2015). Beides kann dazu führen, dass die betroffenen Kraftwerke gedrosselt werden müssen oder weniger effizient arbeiten (Europäische Kommission 2007; Bundesregierung 2008; Kuckshinrichs et al. 2008; Finley und Schuchard 2009; Hirschl und Dunkelberg 2009; Lauwe 2009; Engelhard 2011; Wachsmuth et al. 2012; Bardt et al. 2013), da ein größerer Teil der erzeugten Energie für das Pumpen eingesetzt werden muss (Eskeland et al. 2008). Aufgrund beider Effekte sind die Kraftwerke an Oberläufen im Vergleich zu den Kraftwerken an Flussunterläufen als weniger stark betroffen anzusehen (Engelhard 2011). Sowohl Bardt et al. (2013) als auch Engelhard (2011) gelangen zu dem Ergebnis, dass die Kühlung künftig von der Verfügbarkeit von Flusswasser unabhängiger werde, da die Kraftwerke zunehmend über Kühltürme verfügten. Hirschl und Dunkelberg (2009) sehen die Effizienz der Kühltürme insbesondere bei hohen Temperaturen allerdings ebenfalls beeinträchtigt.

Die Produktion innerhalb thermischer Kraftwerke wird unter Klimawandelgesichtspunkten zusammenfassend somit vor allem von den hydrologischen Standortgegebenheiten beeinflusst, also vom Abfluss und der Wassertemperatur. Daneben sind die Wahl des Kühlsystems und der vom Kühlsystem abhängige Kühlwasserbedarf von Bedeutung. Einfache Durchlaufkühlsysteme sind gegenüber hohen Wassertemperaturen und einer geringen Wasserverfügbarkeit - beispielsweise bei niedrigen Pegelständen - anfälliger als Kreislaufkühlsysteme (Hoffmann et al. 2012; Koch und Vögele 2013; Koch et al. 2014).

\section{Regenerative Energieträger}

Bei der Windkraftnutzung muss zwischen der On- und OffshoreErzeugung unterschieden werden (Cortekar und Groth 2013). So sind die Onshore-Anlagen wesentlich durch eine mögliche Zunahme von Starkwinden in zweierlei Hinsicht beeinträchtigt: Einerseits steigen die Anforderungen an die Standfestigkeit und die mechanische Belastung der Bauteile, andererseits könnten Zwangsabschaltungen häufiger notwendig werden (Hirschl und Dunkelberg 2009; Lauwe 2009; Scheele und Oberdörffer 2011; Bardt et al. 2013). An Offshore-Anlagen werden zusätzliche Anforderungen hinsichtlich der Standfestigkeit, beispielsweise aufgrund des steigenden Meeresspiegels, steigender Wellenhöhen und einer möglichen Zunahme von Sturmfluten gestellt (Hirschl und Dunkelberg 2009; Scheele und Oberdörffer 2011).

Hinsichtlich möglicher Auswirkungen des Klimawandels auf das Potenzial der Windkraftnutzung sind bis Mitte des Jahrhunderts nur geringe Einflüsse zu erwarten. Für Nord- und Zentraleuropa ist für Onshore-Windkraft mit positiven Effekten zu rechnen - vor allem im Herbst und Winter (Pryor et al. 2005a, 2005b). Hinsichtlich des Potenzials von Offshore-Windanlagen ist in Nordeuropa eine geringfügige Abnahme zu erwarten, wobei hier eine große Streuung der Ergebnisse zu berücksichtigen ist (Barstad et al. 2012). In der Summe ist ein positiver Nettoeffekt der Auswirkungen des Klimawandels zu erwarten, wobei es zu monatlichen Unterschieden kommen kann. Innerhalb Deutschlands sind für Mittel- und Norddeutschland eher positive Effekte zu erwarten, wohingegen in Süddeutschland lediglich geringe positive bzw. mitunter auch negative Effekte möglich sind. Für die Jahresproduktion ergibt sich insgesamt eine Erhöhung um 2-5\% (Koch et al. 2015).

Die Erzeugungsanlagen zur Nutzung der Sonnenenergie werden allgemein als robust bzgl. klimatischer Veränderungen eingeschätzt. Weder Photovoltaikanlagen noch solarthermische Kraftwerke werden in ihrem Betrieb durch höhere Temperaturen beeinträchtigt (Cortekar und Groth 2013). Eine geringe Anfälligkeit zeigt sich lediglich bei Wetterextremen wie Starkwinden, Hagel und Blitzschlag, wodurch die Anforderungen an die Sicherheit der Befestigung z. B. auf Hausdächern steigen können (Bundesregierung 2008; Scheele und Oberdörffer 2011; Bardt et al. 2013). Eskeland et al. (2008) weisen darauf hin, dass die Effizienz von Photovoltaikanlagen bei hohen Temperaturen und insbesondere bei länger anhaltenden Hitzewellen geringfügig reduziert sein kann. Für Photovoltaikanlagen wurden die 
Ein weiteres Gefährdungspotenzial geht von Hochwasserereignissen aus. So könnten Mastfundamente unterspült werden, was veränderte Anforderungen an die Standfestigkeit mit sich bringen würde, und Umspannungsanlagen von Hochwasser zunehmend durch Überflutung betroffen sein (Europäische Kommission 2007; Bundesregierung 2008; Groth und Cortekar 2015).

Wenngleich die Überlandverkabelung besonders exponiert gegenüber klimatischen Einflüssen ist, so gibt es vereinzelt auch Gefährdungspotenziale für Erdkabel. So könnten durch Hochwasser Kabeltrassen freigespült werden (Dunkelberg et al. 2009; Hirschl und Dunkelberg 2009) oder in langen Hitzeperioden Schäden an Erdkabeln entstehen (Kuckshinrichs et al. 2008; Hirschl und Dunkelberg 2009). Wachsmuth et al. (2012) weisen allerdings darauf hin, dass noch keine Wirkmodelle vorliegen, die ein verändertes Ausfallverhalten im Zusammenhang mit einer Veränderung von Klima- oder Wetterparametern (Bodentemperatur und -feuchte) erklären können. Anlagen, und selbst dort ist der Kühlwasserbedarf deutlich geringer als bei anderen thermischen Kraftwerken. Demgegenüber ist zu erwarten, dass vor allem der Ertrag aus Biomasse von den Folgen des Klimawandels beeinflusst wird, da die Bodenbeschaffenheit kaum geschützt werden kann (Bardt et al. 2013).

Auf die Gewinnung von Energie aus Erdwärme (Geothermie) sind für Deutschland keine spürbaren Auswirkungen durch den Klimawandel zu erwarten - diese Einschätzung bezieht sich einerseits auf den Betrieb der Anlagen, andererseits aber auch auf Ertragsschwankungen (Cortekar und Groth 2013).

\subsubsection{Auswirkungen des Klimawandels auf die Energieübertragung und -verteilung}

Der Wertschöpfungsstufe Energieübertragung und -verteilung ist die größte physikalische Anfälligkeit zu attestieren (Groth und Cortekar 2015). Davon ist zum einen die physikalische Leitungsfähigkeit bei hohen Temperaturen im Sommer und niedrigen Temperaturen im Winter betroffen (Deb 2000; Stephen et al. 2002; Hu et al. 2006; Eskeland et al. 2008; Dunkelberg et al. 2009; Finley und Schuchard 2009; Hirschl und Dunkelberg 2009; Rothstein und Halbig 2010; Bardt et al. 2013), zum anderen kann die Übertragungsinfrastruktur (Masten, Kabel, Transformatoren) selbst durch Wetterextreme beeinträchtigt bzw. beschädigt werden (Groth und Cortekar 2015).

Dabei zeigen sich deutliche Unterschiede hinsichtlich der Betroffenheit in den unterschiedlichen Netzen. Während die Verteilnetze bereits heute zu einem beträchtlichen Teil unterirdisch verlaufen und somit nur eine geringe Klimaexposition gegeben ist (Engelhard 2011), verlaufen die Übertragungsnetze größtenteils überirdisch und sind den Wetter- und Klimaeinflüssen direkt ausgesetzt. Die Auswirkungen auf den oberirdischen Teil der Netze können dabei vielfältig sein. Wenngleich die Masten und Leitungen grundsätzlich auf Wetterextreme ausgelegt sind, so können sie beispielsweise durch hohe Eis- bzw. Schneelasten oder Blitzschläge beschädigt werden (Bundesregierung 2008; Hirschl und Dunkelberg 2009; Scheele und Oberdörffer 2011). Es ist allerdings wahrscheinlich, dass bisher schon beobachtete Zusammenbrüche der Masten nicht monokausal auf die Eis- und Schneelast zurückzuführen sind, sondern auf das Zusammenwirken unterschiedlicher Einflussfaktoren (Bundesnetzagentur 2006; Lauwe 2009; Schmitt 2012).

\subsubsection{Struktur der Energieversorgung und -infrastruktur in Hamburg}

Der jährliche Gesamtstromverbrauch belief sich im Jahr 2014 in Hamburg auf 12,4 TWh (Energieportal Hamburg 2015). Dabei schwankt die Erzeugungsbilanz in der Regel je nach Tag und Tageszeit.

Ganz überwiegend ist Hamburg jedoch stark von einer Einspeisung von außerhalb der Stadt abhängig. Für das gesamte Jahr 2014 wurde der Gesamtstromverbrauch beispielsweise nur im Umfang von 2,9 TWh durch in Hamburg erzeugten Strom gedeckt, was einem Anteil von rund $23 \%$ entspricht.

Basierend auf den jüngsten Daten des Statistischen Amtes für Hamburg und Schleswig-Holstein (2014) zur Nettostromerzeugung in Hamburg wird deutlich, dass wiederum 81,6 \% des in 2013 in Hamburg erzeugten Stroms aus fossilen Energieträgern, 14,8 \% aus erneuerbaren Energiequellen, 1,2 \% aus nichtbiogenen Abfällen und 2,5 \% aus sonstigen Energieträgern (inkl. Pumpspeicheranlagen) stammte.

Im Hinblick auf den Anteil erneuerbarer Energien zeigt sich für Hamburg zum 31.12.2014 die folgende Struktur und Menge der installierten Leistung (Energieportal Hamburg 2015): Insgesamt belief sich die installierte Leistung regenerativer Energien auf $144.083 \mathrm{~kW}$, bereitgestellt von 2910 Anlagen. Davon entfielen auf Solarenergie $34.733 \mathrm{~kW}$ (2653 Anlagen), auf Windenergie $59.340 \mathrm{~kW}$ (59 Anlagen), auf Wasserkraft 0,110 kW (1 Anlage) sowie auf Biomasse 49.900 kW (197 Anlagen). Deponie-, Klärund Grubengas wurden in Hamburg nicht genutzt.

Es ist wichtig, neben der Struktur der Energieversorgung in Hamburg auch die infrastrukturellen Gegebenheiten des Stromnetzes zu betrachten. Deutschlandweit sind insgesamt vier Übertragungsnetzbetreiber dafür zuständig, den Strom zu rund 900 Verteilnetzbetreibern zu transportieren. Für die Metropolregion und das Bundesland Hamburg gibt es unterschiedliche Betreiber. So ist für das Bundesland Hamburg die $50 \mathrm{~Hz}$ Transmission $\mathrm{GmbH}$ zuständig, für die Metropolregion jedoch die Tennet TSO $\mathrm{GmbH}$. Die elektrische Energie kommt mit 380 kV Höchstspannung über Höchstspannungsleitungen nach Hamburg. Von drei 
Umspannwerken, die an das Übertragungsnetz der 50 Hz Transmission $\mathrm{GmbH}$ angeschlossen sind, wird die elektrische Energie dann von 380 auf $110 \mathrm{kV}$ umgewandelt und in das Hamburger Verteilnetz eingespeist. In insgesamt 53 Umspannwerken wird der Strom von der Hochspannung auf die Mittelspannung heruntertransformiert und gelangt anschließend über rund 7500 Kunden- und Netzstationen in das Stromnetz der Niederspannungsebene (Stromnetz Hamburg GmbH 2015).

Eine spezifische Übersicht zum aktuellen Zustand der Energieinfrastruktur fasst die technischen Daten des Stromverteilnetzes der Hansestadt zusammen (Stromnetz Hamburg GmbH 2014). Da der dort zitierte Netzzustandsbericht 2014 und der Strukturbericht 2014 nur der Bundesnetzagentur zur Verfügung gestellt werden und somit nicht öffentlich verfügbar sind (Polkehn-Appel 2015), ist dies hierzu die einzige verfügbare Quelle. Die nachfolgenden Ausführungen zur Struktur der Energieinfrastruktur in Hamburg beziehen sich daher ausschließlich auf diese Übersicht.

Das Verteilnetz besteht aus Teilnetzen in den Spannungsebenen Hochspannung $(110 \mathrm{kV})$, Mittelspannung $(10 \mathrm{kV})$ und Niederspannung $(0,4 \mathrm{kV})$. Zum Stromverteilnetz in Hamburg gehören über $27.000 \mathrm{~km}$ Stromleitungen, wobei $44 \%$ der Leitungen bereits vor 1970 verlegt bzw. errichtet wurden. 95 \% der Stromkabel sind unterirdisch verlegt. Die Stromkreislängen betragen für die Hochspannung 362 km als Kabel in der Erde und 613 km als Freileitungen. Die Stromkreislänge der Mittelspannung besteht ausschließlich aus unterirdisch verlegten Kabeln mit einer Gesamtlänge von $5833 \mathrm{~km}$. Im Bereich der Niederspannung werden $19.958 \mathrm{~km}$ unterirdisch verlegte Kabel und $731 \mathrm{~km}$ Freileitungen genutzt. Die Endverbraucher in Hamburg teilen sich ungefähr in 29 Hochspannungskunden, 2000 Mittelspannungskunden und 1,1 Mio. Niederspannungskunden auf.

Insgesamt sind die Anlagen des Verteilnetzes derzeit in einem anforderungsgerechten Zustand, wobei sich das Alter der Anlagen deutlich unterscheidet. Durch die zunehmende Alterung der Anlagen mit einer betriebsgewöhnlichen Nutzungsdauer zwischen 30 und 40 Jahren ist jedoch bereits jetzt ein Bedarf für Sanierungen oder sogar den Ersatz von Anlagen gegeben, beispielsweise bei Hochspannungs- und Mittelspannungsschaltanlagen in den Umspannwerken.

Mittelfristig wird Sanierungsbedarf u. a. auch bei Mittelspannungskabeln, Transformatoren im Bereich Hochspannung zu Mittelspannung oder auch Hochspannungsschaltanlagen in den Umspannwerken gesehen.

Der Bereich der Hochspannungsfreileitungen und -kabel besteht zu rund zwei Dritteln aus Freileitungen, wobei die Masten ausschließlich aus Stahl gefertigt sind. Bei den Freileitungen sind $97 \%$ und bei den Kabeln $82 \%$ älter als 20 Jahre. Mittelfristiger Sanierungsbedarf wird im Bereich der Kabel gesehen. Im Bereich der Freileitungen und Masten ist erst langfristig Sanierungsbedarf zu erwarten.

Hinsichtlich der Anlagen auf der Umspannebene von Hochzu Mittelspannung ist entsprechend der Altersstruktur - $78 \%$ der Transformatoren und $28 \%$ der Umspannwerke wurden vor 1991 in Betrieb genommen - mittelfristig mit einem stark zunehmenden Sanierungsbedarf bei den Transformatoren zu rechnen.

Auch für Freileitungen und Kabel im Bereich der Niederspannung ist mittelfristig mit einem Anstieg des Sanierungs- bedarfs zu rechnen, da entsprechend der Altersstruktur in den kommenden Jahren zunehmend viele Niederspannungskabel die betriebsgewöhnliche Nutzungsdauer von 40 Jahren erreichen werden. In Hamburg betrifft dies rund $8000 \mathrm{~km}$ der Kabel, die bereits vor 1970 gelegt wurden. Der Bestand an Freileitungen mit meist Holzmasten befindet sich in Kleingartenanlagen und Stadtrandsiedlungen, wobei Freileitungen nicht mehr neu errichtet werden.

\subsubsection{Mögliche Auswirkungen des Klimawandels auf die Energieversorgung und -infrastruktur in Hamburg}

Bislang liegen noch keine wissenschaftlichen Untersuchungen vor, die sich umfassend mit der Analyse des Klimawandels und seinen Auswirkungen auf die Energieversorgung in Hamburg und der Metropolregion beschäftigt haben. Auch in den von der Stadt Hamburg veröffentlichten Konzepten zum Klimaschutz oder zur Anpassung an die Folgen des Klimawandels werden diese Aspekte, wenn überhaupt, nur kurz adressiert. Im Masterplan Klimaschutz (Bürgerschaft der Freien und Hansestadt Hamburg 2013a) wird im Bereich Energie/Energieversorgung insbesondere auf geplante Aktivitäten zur Einsparung von Energie, Effizienzsteigerungen sowie den Ausbau und die Integration erneuerbarer Energien eingegangen. Detaillierte Notwendigkeiten im Hinblick auf den Ausbau oder die Instandsetzung von Energieinfrastrukturen werden dort ebenso wenig thematisiert wie die Rolle möglicher Klimawandelfolgen. Innerhalb des Aktionsplans Anpassung an den Klimawandel (Bürgerschaft der Freien und Hansestadt Hamburg 2013b) wird einleitend die Zunahme von Extremwetterereignissen als eine besonders relevante Auswirkung des Klimawandels angesprochen und als hier wichtige vorbereitende Maßnahme die Katastrophenschutzplanung für Stromausfälle genannt. Des Weiteren wird nur noch im Rahmen des Handlungsfeldes Infrastrukturen auf die Energieversorgung eingegangen, indem die langfristige Sicherstellung der Energieversorgung als ein Ziel der Anpassung genannt wird - Maßnahmen dazu werden jedoch keine benannt. Im Zuge der Berichterstattung der Stadt Hamburg innerhalb des Cities Program des CDP (ehemals Carbon Disclosure Project; CDP Worldwide 2014a) unter Nutzung des CDP Cities 2014 Information Request (CDP Worldwide 2014b) werden zwar unterschiedliche klimawandelbedingte Risiken und mögliche Auswirkungen auf Hamburg genannt, die Energieversorgung wird jedoch nicht angesprochen (CDP Worldwide 2014c).

Der in Hamburg erzeugte Strom basiert vor allem auf fossilen Energieträgern. Zukünftig ist hier vor dem Hintergrund der Klimaschutzziele im Rahmen der Energiewende ein weiterer Ausbau des Anteils erneuerbarer Energien zu erwarten. Durch diese weitere Dezentralisierung des Versorgungssystems und die damit verbundene stärkere Bedeutung regenerativer Energiequellen verändert sich auch die Anfälligkeit des Systems gegenüber Klimaänderungen, sowohl was das Zusammenspiel einer größeren Zahl kleinerer Kraftwerke angeht als auch die größeren Anforderungen an die Netzinfrastrukturen zum Stromtransport. 
Deutlich ist beispielsweise, dass sich auf der einen Seite die Abhängigkeiten von langen Transportketten und den Gewinnungsbedingungen in den Rohstofflieferländern verringern. Auf der anderen Seite sind die regenerativen Energiequellen deutlich abhängiger von den vorherrschenden und variablen Wetterbedingungen: Sonnenscheindauer, Windstärken, Wolkenbildung und Wasserdargebot können die Leistungsfähigkeit dieser Energieformen beeinträchtigen, aber auch steigern.

In den bisherigen Ausführungen zur Struktur der Energieversorgung ist deutlich geworden, dass Hamburg überwiegend von einer Einspeisung von außerhalb abhängig ist. Zudem sind Teile des Verteilnetzes relativ alt, sodass hier auch bereits kurz- bis mittelfristig Sanierungsbedarf zu erwarten ist. Somit sind insbesondere mögliche klimawandelbedingte Auswirkungen auf die Energienetzinfrastruktur relevant. Diese werden nachfolgend betrachtet.

Bislang wurden für Hamburg lediglich als Folge der schneeund eisbedingten Schäden im Münsterland im Winter 2005 Prüfungen zum Erfordernis von Sanierungsmaßnahmen vorgenommen. Bei dem extremen Wetterereignis am 25. Dezember 2005 war es zu ungewöhnlich starken Schneefällen gekommen. Daraufhin waren beispielweise in den Landkreisen Steinfurt und Borken 50 Strommasten unter der Schneelast zusammengebrochen und in der Folge bis zu 600.000 Menschen von der Stromversorgung abgeschnitten (Bundesnetzagentur 2006; Schmitt 2012). In dieser Extremsituation hatte das Zusammentreffen unterschiedlicher Faktoren die Masten deutlich stärker beansprucht, als es die Errichtungsnorm vorsieht, was zu der umfassenden Schadenssituation geführt hatte (Bundesnetzagentur 2006). Einflussfaktoren waren:

1. starker Wind (bis Orkanstärke), der das Anbacken des Schnees erst ermöglichte und Staudruck auf die Leiterseile ausübte,

2. extreme Schneefallmengen,

3. Temperaturen um $0{ }^{\circ} \mathrm{C}$,

4. sehr nasser Schnee mit hohem spezifischem Gewicht,

5. einsetzender Regen, der die Schnee- und Eiswalzen weiter beschwerte,

6. eine einseitige Belastung der Abspannfelder, die auf Abspannmasten torsionsauslösend wirkten,

7. eine Windrichtung senkrecht zur Trassenführung sowie

8. einzelne Leiterseile, die in sich drehbar waren und das Anwachsen auf allen Seiten ermöglichten.

Für Hamburg ergaben Prüfungen im Hinblick auf Wind- und Eisbelastungsklassen sowie andere Bestimmungen keinen Befund. Die Studie wurde durch die LBD-Beratungsgesellschaft durchgeführt, ist aber nicht öffentlich verfügbar (hier zitiert nach Stromnetz Hamburg GmbH 2014).

Grundsätzlich ist zu berücksichtigen, dass das Verteilnetz in Hamburg nicht nur teilweise relativ alt ist und zukünftig ohnehin in unterschiedlichen Fristen verschiedene Sanierungs- und Instandhaltungsmaßnahmen durchzuführen sind, sondern dass es auch strukturelle Besonderheiten aufweist, die zu klimawandelbedingten Betroffenheiten führen können.

Aufgrund der geographischen Lage Hamburgs mit Zugang zur Nordsee sind große Teile des Stadtgebietes und der Metropolregion sturmflutgefährdet (s. dazu das einführende $>$ Kap. 4 dieses Klimaberichtes), was bei der Planung von Standorten und
Trassen berücksichtigt werden sollte. Auch ist die Wahlmöglichkeit hinsichtlich der Leitungstrassen aufgrund einer überdurchschnittlichen Anzahl an Wasserüberquerungen erheblich eingeschränkt (Stromnetz Hamburg GmbH 2014).

In welcher Form ganz konkret klimawandelbedingte Auswirkungen für die Energieversorgung und -infrastruktur in Hamburg zu erwarten sind, ist nach aktuellem Wissensstand nicht zu beantworten. Jedoch ist davon auszugehen, dass die innerhalb dieses Kapitels für Deutschland beschriebenen Auswirkungen des Klimawandels für die Energieübertragung und -verteilung grundsätzlich auch für Hamburg zu erwarten sind. Positiv wirkt sich aus, dass 95 \% der Stromkabel im Hamburger Stromnetz unterirdisch verlegt sind. Somit besteht hier bereits ein Schutz vor äußeren Einflüssen wie Extremwetterereignissen oder Blitzeinschlägen.

Der Gefahr eines umfassenden Stromausfalls hat Hamburg entgegenzusetzen, dass durch die Einbindung der Anlagen in sog. Versorgungsringe immer eine Versorgung von zwei Seiten möglich ist. Bisherige Untersuchungen zur Versorgungszuverlässigkeit des Hamburger Stromnetzes für das Jahr 2014 zeigen, dass ein Hamburger Netzkunde im Jahr durchschnittlich 11,7 min von einem Stromausfall betroffen war. Die durchschnittliche Dauer eines Stromausfalls betrug 60,6 min. Im Durchschnitt muss jeder Hamburger Niederspannungskunde alle 5,2 Jahre mit einem Stromausfall rechnen (Stromnetz Hamburg GmbH 2015). Die durchschnittliche Länge der Versorgungsunterbrechungen je Stromverbraucher in Deutschland belief sich demgegenüber im Jahr 2013 beispielsweise auf 15,32 min (Bundesnetzagentur 2014).

Um ein hohes Maß an Versorgungssicherheit aufrechtzuerhalten, bietet es sich an, dass auch in Hamburg die ohnehin anstehenden Sanierungs- und Instandhaltungsmaßnahmen durch die ergänzende Berücksichtigung regionaler Klimainformationen so dimensioniert werden, dass sie auch zum Beseitigen potenzieller Schwachstellen genutzt werden.

\subsubsection{Zwischenfazit}

Von den möglichen Auswirkungen des Klimawandels wird eine Vielzahl gesellschaftlicher Bereiche betroffen sein, wobei dem Energiesektor eine große Bedeutung zukommt. Basierend auf der Auswertung des aktuellen Wissensstandes zu klimawandelbedingten Betroffenheiten des Energiesektors zeigt sich, dass die meisten Bereiche der Wertschöpfungskette negativ betroffen sein werden. Als wesentliche Einflussfaktoren konnten die Wasserverfügbarkeit, Extremwetterereignisse und steigende Durchschnittstemperaturen identifiziert werden. Zudem ist deutlich geworden, dass die Netzinfrastruktur die verletzlichste Komponente des Energiesystems ist. Auch wenn sich bei einzelnen Aspekten unterschiedliche Einschätzungen gezeigt haben, so kann doch festgehalten werden, dass die Betroffenheit der Energieversorgung insgesamt als grundsätzlich gut handhabbar anzusehen ist.

Darüber hinaus lassen sich die Auswirkungen des Klimawandels jahreszeitlich differenzieren. Extremwetterbedingte Schäden an Kraftwerken und Leitungsnetzen sind vorranging in den Wintermonaten zu erwarten, während im Sommer Versorgungsengpässe infolge einer Zunahme des Kühlenergiebedarfs bei gleich- 
zeitiger Beeinträchtigung der Stromproduktion bei Wasser- und thermischen Kraftwerken auftreten können.

Es ist deutlich geworden, dass die zu erwartenden Auswirkungen des Klimawandels schon jetzt auch in Hamburg und der Metropolregion für anstehende Infrastrukturmaßnahmen relevant sind. Um damit verbundene negative Auswirkungen $\mathrm{zu}$ vermeiden oder zumindest abzumildern, besteht beispielsweise die Möglichkeit, die ohnehin notwendige Transformation der Energiesysteme auch zum Beseitigen entsprechender Schwachstellen zu nutzen. Zudem haben Investitionen in Infrastrukturen wie Anlagen zur Energieerzeugung oder Netze in der Regel sehr langfristige Konsequenzen und Nutzungsdauern von 40 Jahren oder mehr. Selbst durchschnittliche Technologieerneuerungszyklen bei kostenintensiven Bauteilen liegen noch bei rund 30 Jahren. Einmal getätigte (Fehl-)Investitionen können also nur in langen Zeiträumen oder zu hohen Kosten korrigiert werden (Groth und Cortekar 2014).

Wie sich in diesem Teilkapitel jedoch auch gezeigt hat, bestehen im Hinblick auf spezifische Aussagen zu Betroffenheiten der Energieversorgung in Hamburg und der Metropolregion noch Wissenslücken und Forschungsbedarf. Dies liegt insbesondere daran, dass dazu bislang regional keine spezifischen wissenschaftlichen Untersuchungen durchgeführt wurden. Auch erfolgt in diesem Kapitel eine Fokussierung auf die Stromversorgung, sodass beispielsweise für die Wärme- und die Gasversorgung keine Aussagen getroffen werden können. Zudem besteht noch ein umfassender Forschungsbedarf zu KostenNutzen-Abschätzungen von Anpassungsmaßnahmen im Energiesektor. Dies gilt auch für die notwendige Berücksichtigung von Klimawandelfolgen im Rahmen der zukünftigen Ausgestaltung regulatorischer Rahmenbedingungen sowie im Hinblick auf die damit verbundenen ökonomischen Anreizsetzungen. Im Mittelpunkt steht hier die Analyse des politischen Rahmens der Energiewende hinsichtlich der Berücksichtigung von Klimawandelfolgen und der Erarbeitung von Handlungsempfehlungen - insbesondere im Hinblick auf die Anpassung von Infrastrukturen der Energieversorgung. Und nicht zuletzt erfordert auch die Berücksichtigung des Einflusses des nationalen und europäischen Energiesystems auf die Energieversorgung regionaler Metropolen weitere wissenschaftliche Analysen. Die entscheidende Auswirkung des Klimawandels auf die Energiewirtschaft besteht derzeit somit insgesamt im regulatorischen Bereich (Groth und Cortekar 2014; Buth et al. 2015). Zudem ist hervorzuheben, dass über die hier vorgenommene isolierte Betrachtung einer einzelnen Versorgungsinfrastruktur hinausgehend weitere Forschungsnotwendigkeiten bestehen. Diesbezüglich ist insbesondere eine erweiterte Systembetrachtung notwendig, die sowohl die Wärme- und Gasversorgung als auch mögliche Wechselwirkungen grundlegend unterschiedlicher Infrastrukturen beinhaltet. $\mathrm{Zu}$ nennen ist hier beispielsweise die Relevanz von Kaskadeneffekten Kritischer Infrastrukturen wie der Energie- und Wasserversorgung.

Des Weiteren besteht für Deutschland sowie spezifisch für die Metropolregion Hamburg Forschungsbedarf dahingehend, welche klimawandelbedingten Auswirkungen auf die Stromnachfrage zu erwarten sind und wo dort zukünftig mit kritischen Spitzenlasten zu rechnen ist. Vor dem Hintergrund des im Rahmen der Energiewende notwendigen Strukturwandels der Energieversorgung in Deutschland wurden diese Aspekte hier ausgeklammert. Die grundsätzliche Relevanz auch der Betrachtung von Nachfrageänderungen ist ohne Frage vorhanden und wird auch in der Literatur entsprechend betont (U.S. - Canada Power System Outage Task Force 2004; Jayant et al. 2011).

\subsection{Wasserversorgung}

\subsubsection{Stand des Wissens zu klimawandelbedingten Betroffenheiten der Wasserversorgung und -infrastruktur in Deutschland}

\subsubsection{Auswirkungen des Klimawandels auf Wasserangebot, Wasserversorgungs- infrastruktur und Abwasserentsorgung}

\section{Hoch- und Niedrigwasser}

In Einzugsgebieten von Flüssen wirken sich vor allem Hochund Niedrigwasserereignisse sowie damit einhergehende Veränderungen des Grundwasserspiegels auf die Wassernutzung aus. Sinkt der mittlere Niedrigwasserabfluss unter bestimmte Schwellenwerte, ist mit einer Beeinträchtigung der Wasserversorgung für Wärmekraftwerke, Bergbau, verarbeitendes Gewerbe sowie Landwirtschaft und der öffentlichen Wasserversorgung zu rechnen. Da Niedrigwasser im Zuge langer Dürreperioden auch zu einem Absinken des Grundwasserspiegels führt, kann es zu Problemen bei der Gewinnung von Trinkwasser kommen, die in vielen Regionen Deutschlands aus dem Grundwasserspeicher erfolgt (Scherzer et al. 2010). Dannenberg et al. (2009) erwarten, abgesehen von lokalen und temporären Einzelereignissen, in Deutschland allerdings keine zukünftige Gefährdung der Trinkwassersicherheit.

Neben Trockenperioden werden im Zuge des Klimawandels auch verstärkt Starkniederschläge erwartet, die zu Hochwasserereignissen führen können (Braubach 2011). Eine Untersuchung des Augusthochwassers 2002 in Sachsen und Sachsen-Anhalt hat gezeigt, dass durch ein solches Ereignis erhebliche Schäden an der Wasserversorgungsinfrastruktur entstehen können und die Wasserqualität beeinträchtigt werden kann. In $66 \%$ der Kreise mit hochwasserbetroffener Wasserversorgung war die Versorgung eingeschränkt, und in 50 \% dieser Kreise kam es zu Beeinträchtigungen der Wasserqualität (Braubach 2011). Die Beeinträchtigung der Wasserversorgung wurde dabei durch Schäden an Anlagen der Energie-, Mess-, Steuer- und Regelungstechnik, an Brunnen- und Fassungsanlagen, an Wasseraufbereitungsanlagen sowie am Rohrnetz verursacht (Wricke et al. 2003).

\section{Veränderte Niederschlagsmengen}

Änderungen der Menge und der Variabilität von Niederschlägen können zum einen Auswirkungen auf die Wasserverfügbarkeit haben. Zum anderen können sich die Anforderungen an das Kanalisationsnetz zur Ableitung von Regenwasser ändern.

Zwei Studien für die USA (Titus 1992) bzw. Kalifornien (Hanemann und Dale 2006) zeigen die ökonomischen Schäden eines Wassermangels für die Wasserversorgung der Bevölkerung durch 
niedrigere Niederschlagsmengen auf. Für Kalifornien ergibt sich dadurch ein Schaden in Höhe von 3,2 Mrd. US-\$ pro Jahr. Für die USA wird zudem ein Preisanstieg durch die Wasserverknappung erwartet. Zusammen mit einem Erlösrückgang in der Landwirtschaft aufgrund von Wasserrationierungen wird für die USA mit klimawandelbedingten Schäden in Höhe von 6-8 Mrd. US-\$ pro Jahr gerechnet. Dies sind 10-12\% der Wasserkosten ohne Berücksichtigung des Klimawandels. Entsprechende Berechnungen liegen für Deutschland derzeit nicht vor.

Welche Auswirkungen der Klimawandel auf die Abwasserinfrastruktur haben kann, zeigen zwei Studien für die EmscherLippe-Region sowie für Helsingborg in Schweden. Für die Emscher-Lippe-Region zeigen Kersting und Werbeck (2013), dass die Jahresschmutzwassermenge aufgrund wirtschaftlicher und demographischer Entwicklungen bis zum Jahr 2030 um 25-30 \% zurückgehen wird. Die durch die Kanalisationssysteme abzuleitende Jahresregenwassermenge unter Berücksichtigung des Klimawandels würde hingegen je nach Szenario um 1,4$10,8 \%$ zunehmen. Flächenentsiegelung und Maßnahmen zur Regenwasserversickerung können dieser Zunahme allerdings entgegenwirken.

Für Helsingborg fanden Semadeni-Davies et al. (2008) auf der Basis eines hydrologischen Modells heraus, dass der Klimawandel zu einer Verschärfung der bestehenden Abwasserprobleme führen kann. Dazu gehört vor allem das Überlaufen der Mischwasserkanäle bei Starkregen, was bereits heute mehrmals im Jahr vorkommt und zu Verunreinigungen im Hafenwasser und in Badegewässern führt. Dabei wurden unterschiedliche Klimaszenarien mittels veränderter Regenwassermengen sowie unterschiedlicher Urbanisierungsszenarien untersucht.

\subsubsection{Auswirkungen des Klimawandels auf die Wassernachfrage}

Die Entwicklung des Wasserbedarfs von Haushalten sowie Industrie und Gewerbe hängt grundsätzlich von vielen Faktoren ab. Hierzu gehören demographische und wirtschaftliche Entwicklungen sowie der Wasserpreis und der Einsatz wassersparender Technologien (Ansmann 2013). Außerdem wird ein Zusammenhang zwischen Temperatur und Wasserbedarf angenommen, sodass auch mit Auswirkungen des Klimawandels auf die Wassernachfrage gerechnet wird.

Für das Untersuchungsgebiet Dresden zeigen Tränckner et al. (2012), dass ein spürbarer Einfluss auf den Wasserverbrauch erst ab einer ortspezifischen Temperaturschwelle von 15 bis $20^{\circ} \mathrm{C}$ erfolgt. Dabei steigt der Temperatureinfluss auf den Wasserverbrauch mit den Tagen ohne Niederschlag. Insgesamt zeigt sich aber, dass Wasserverbrauch und Schmutzwasserabfall vorrangig durch die Bevölkerungszahl erklärt werden.

Ähnliche Analysen wurden für die Emscher-Lippe-Region im KLIMZUG-Projekt dynaklim vorgenommen. Es wird erwartet, dass sich hier die durchschnittliche Trinkwassermenge klimawandelbedingt um 2-3\% erhöhen wird. Wirtschaftliche und demographische Entwicklungen (weniger und ältere Einwohner) wirken diesem Anstieg entgegen, wodurch insgesamt mit einem Rückgang der Trink- und Abwassermenge gerechnet wird. Allerdings wird sich die Variabilität der Mengen deutlich erhöhen. Die Bandbreite der klimabedingten Schwankungen der
Tagesmengen steigt von -8 bis $+18 \%$ auf -7 bis +33 bzw. $29 \%$, je nach angenommenem Klimaszenario (Kersting und Werbeck 2013). Das führt dazu, dass die Spitzenkapazitäten seitens der Wasserversorgungsunternehmen weiterhin aufrechterhalten werden müssen. Kersting und Werbeck (2013) schlussfolgern sogar, dass die Wasserversorgungskapazität erhöht werden müsse, falls sich der klimawandelbedingte Anstieg der Spitzenlast früher auswirken sollte als der sozioökonomisch bedingte Rückgang des durchschnittlichen Wasserverbrauchs.

Im Rahmen des Projektes „Anpassungsstrategien an Klimatrends und Extremwetter und Maßnahmen für ein nachhaltiges Grundwassermanagement (AnKliG)“ wurde für den Raum Südhessen eine Wasserbedarfsprognose für das Jahr 2100 erstellt. Dabei wurde sowohl die Entwicklung des Wasserbedarfs in Normaljahren untersucht als auch die Entwicklung von Bedarfsspitzen, die in ausgeprägten Trockenjahren auftreten (Mikat et al. 2010; Roth et al. 2011). Im Prognosezeitraum bis 2100 wird für Trockenjahre mit einem Zuschlag von $8 \%$ auf den Wasserbedarf in Normaljahren gerechnet. Dieser Zuschlag wird im Vergleich zu den Auswirkungen anderer Faktoren als relativ gering angesehen. Wesentlich relevanter für die Entwicklung des Wasserversorgung seien vielmehr die allgemeine Bedarfsentwicklung sowie die Entwicklung des nutzbaren Grundwassers und der Grundwasserneubildung (Roth et al. 2011).

Vereinzelt existieren auch Studien für andere Länder. So wird beispielsweise für England ein Anstieg des Pro-Kopf-Wasserverbrauchs um 2-5\% in den kommenden 20-50 Jahren erwartet (Downing et al. 2003).

Neben den Auswirkungen des Klimawandels auf den Wasserbedarf insgesamt werden auch Auswirkungen auf die saisonalen Schwankungen der Wassernachfrage erwartet. Heiße Sommer oder ein Anstieg von Hitzetagen können diese Schwankungen der Wassernachfrage verstärken. Auf der einen Seite wird mit einem Rückgang des Pro-Kopf-Verbrauchs durch die Bevölkerung und der Wasserabgabe an Gewerbe und Industrie gerechnet. Dies wird vor allem aufgrund von Sanierung sowie Anschaffung neuer, wassersparender Endgeräte erwartet (Tränckner et al. 2012). Auf der anderen Seite müssen die Versorgungsbetriebe die Kapazitäten für den Spitzenbedarf bei langen Hitze- und Trockenperioden sicherstellen. Hierzu zählt auch das Vorhalten von Löschwasser. Diverse industrielle Sektoren wie die Nahrungsmittel- und Getränkeproduktion verzeichnen in den Sommermonaten eine erhöhte Nachfrage. Dem entsprechend steigt auch der Wasserbedarf. Da viele Produkte einen großen Anteil an Wasser enthalten, können technologische Maßnahmen zum Wassersparen in der Produktion nur einen Teil des steigenden Bedarfs ausmachen (Koch und Grünewald 2011).

Der Großteil der veröffentlichten Untersuchungen geht davon aus, dass in den meisten deutschen Regionen mit einem Anstieg des Wasserbedarfs während langer Hitzeperioden zu rechnen ist. Einen Anhaltspunkt für mögliche zukünftige Auswirkungen des Klimawandels geben auch Werte aus anderen Ländern. So lag der Wasserverbrauch im August des Hitzesommers 2003 in den Niederlanden um $15 \%$ über dem langjährigen Mittelwert (EEA 2007). Die Folge solcher Schwankungen können Unterauslastungen von Anlagen in Perioden mit relativ geringem Wasserbedarf sein, die zu Ineffizienzen des Betriebs von Einrichtungen 
führen (Der Beauftragte der Bundesregierung für die Neuen Länder 2011).

\subsubsection{Struktur der Wasserversorgung und Wasserversorgungsinfrastruktur in Hamburg}

\subsubsection{Wasserversorgungs- und Abwasserentsorgungsinfrastruktur} Trinkwasserversorgung

Das Hamburger Verbundsystem besteht aus insgesamt 16 Wasserwerken, welche die Hansestadt Hamburg sowie 20 Umlandgemeinden - das sind rund 2 Mio. Einwohner - mit Trinkwasser versorgen (HAMBURG WASSER 2015b). Das Betriebsgelände im Stadtteil Rothenburgsort bildet das Kernstück der Trinkwasserversorgung in Hamburg. Von hier aus werden alle Wasserwerke des Verbunds gesteuert und überwacht. Es besteht aus dem Hauptpumpwerk, von dem mehr als $25 \%$ des Trinkwassers in das Verteilnetz abgegeben werden, sowie dem Wasserwerk Billbrook und einem Wasserlabor. Außerdem befinden sich hier die Verwaltung sowie Konferenz-, Schulungs- und Ausbildungszentren (HAMBURG WASSER 2015c).

Das Grundwasser wird mittels Brunnen gefördert. Die meisten Brunnen im Hamburger Verbund sind Vertikalbrunnen, die in Tiefen von 20 bis $429 \mathrm{~m}$ reichen. Unterhalb des Grundwasserspiegels eingebaute Unterwasserpumpen fördern das Wasser zum Wasserwerk. Des Weiteren kommen Flachbrunnen zum Einsatz, die bis maximal $24 \mathrm{~m}$ in die Tiefe reichen. Das Wasser wird hier mittels Vakuumpumpen in die Wasserwerke transportiert. In den Wasserwerken wird das Wasser aufbereitet und gefiltert und anschließend in große Reinwasserbehälter geleitet. Von hier aus wird es je nach Bedarf mittels Pumpen durch das Versorgungsnetz zum Verbraucher transportiert. Um die Wasserleitungen vor Bodenfrost zu schützen, sind sie ca. 1,5 m unter der Oberfläche verlegt. Das Anlagevermögen von HAMBURG WASSER, das für die Wasserversorgung eingesetzt wird, beläuft sich auf 521 Mio. $€$ (HAMBURG WASSER 2015a).

Die Trinkwasserbehälter dienen auch als Puffer für die ständig schwankende Wasserentnahme. Sie leeren sich bei hohem Bedarf (tagsüber) und füllen sich bei geringem Bedarf (nachts). Damit auch in Notfällen (z. B. beim Ausfall eines Wasserwerks) die Versorgung sichergestellt ist, lässt sich das Wasser innerhalb des Verbunds umleiten, da jede größere Verbrauchszone von mehreren Wasserwerken beliefert wird. Bei geringem Verbrauch in der Nacht werden einige Werke auch gänzlich abgeschaltet. Damit Wasser bei Bedarf von einer in die andere Zone abgegeben werden kann, sind z. T. Pumpen oder regelbare Armaturen installiert, denn der Wasserdruck ist je nach Höhenlage in den Zonen unterschiedlich. Teilweise werden auch Hochbehälter in Höhenlage (z. B. in Baursberg) eingesetzt, um den Wasserdruck aufrechtzuerhalten. Dieser liegt im Hamburger Gebiet zwischen 2,0 und 7,0 bar.

Die Verfügbarkeit nutzbaren Grundwassers ist in Hamburg also Grundlage für eine sichere Trinkwasserversorgung. Durch die starke Versiegelung durch Gebäude, Straßen und Plätze in der Stadt Hamburg (wie auch in anderen urbanen Räumen) ergeben sich allerdings Probleme bei der Grundwasserneubildung. Der natürliche Kreislauf, bei dem ein Teil des Regenwassers versickert und Grundwasser bildet, wird gestört, da der Niederschlag vermehrt über Oberflächengewässer abfließt. Zudem haben Eindeichung, Begradigung und Vertiefung von Flüssen, die Trockenlegung von Mooren und das Abholzen von Wäldern den Grundwasserspiegel bereits vielerorts sinken lassen.

\section{Abwasserentsorgung}

Das Abwassernetz wird in Hamburg Sielnetz genannt. Es bezeichnet das System unterirdischer Rohre und Kanäle, über die das städtische Abwasser zum Klärwerk geleitet wird. Insgesamt besteht dieses System aus $5800 \mathrm{~km}$ Leitungen - davon sind ca. ein Viertel Mischsiele (Regen- und Schmutzwasser fließen hier gemeinsam). Der Rest des Abwassers wird über eine Trennkanalisation abgeleitet. Hier werden Schmutz- und Regenwasser unabhängig voneinander geleitet. Das Schmutzwasser wird zum Klärwerk transportiert und dort gereinigt. Das Regenwasser wird in umliegende Gewässer geleitet oder in der Region versickert. Dies fördert die Grundwasserneubildung und hält mehr Wasser in der Region. Insgesamt gibt es vier Klärwerke. Nahezu das gesamte Abwasser aus Hamburg und einigen Randgebieten wird in den Klärwerksanlagen Köhlbrandhöft und Dradenau behandelt. Sielnetze, Klärwerke und Pumpanlagen bildeten im Jahr 2013 zusammen ein Anlagevermögen von 3,193 Mrd. € (HAMBURG WASSER 2015d).

Besonders bei starken Regenfällen muss die örtliche Kanalisation entlastet werden, um sie vor dem Überlaufen in Grundstücke und in die Gewässer zu schützen. Hierfür dienen Transportsiele, Sammler und Mischwasser-Rückhaltebecken als Speicher. Sind die Siele voll, läuft das Wasser in die Rückhaltebecken, wo es zwischengespeichert und bei Wiederaufnahmefähigkeit der Siele zurückgepumpt wird.

Insbesondere die im Zuge der wachsenden Stadt Hamburg fortschreitende Versiegelung verhindert die Versickerung der Niederschläge. Dies führt bei starkem Regen zu einer Überlastung der Abwasserleitungen. Durch die Entlastung der Systeme soll möglichst ein Überlaufen der Mischkanalisation in die Oberflächengewässer verhindert werden, was wiederum eine starke Belastung für Flora und Fauna bedeutet.

\section{Wasserabgabe}

Die Wasserabgabe der Hamburger Wasserwerke betrug im Jahr 2011 rund 108,8 Mio. $\mathrm{m}^{3}$, die sich wie folgt auf die einzelnen Verbrauchsgruppen verteilt (Kluge et al. 2014):

$\begin{array}{lll}\text { Haushalte } & 75,8 \text { Mio. }^{3} & (69,7 \%) \\ \text { Gewerbe, Handel, Dienstleistungen } & 23,3 \text { Mio. } \mathrm{m}^{3} & (21,4 \%) \\ \text { Weiterverteiler } & 3,5 \mathrm{Mio}^{3} & (3,1 \%) \\ \text { Industrie } & 2,8 \mathrm{Mio} \cdot \mathrm{m}^{3} & (2,6 \%) \\ \text { Sonstige } & 1,6 \text { Mio. } \mathrm{m}^{3} & (1,5 \%)\end{array}$

Die größte Abnehmergruppe der Hamburger Wasserwerke sind demnach die Haushalte, die knapp $70 \%$ des Wassers erhalten. Rund $21 \%$ der Wasserabgabe entfallen auf Gewerbe, 
Handel und Dienstleistungen. Industriebetriebe nehmen lediglich 2,6\% des Wassers ab. Die Wasserentnahme für die Produktionsprozesse erfolgt vorwiegend aus der Natur (Statistisches Bundesamt 2014).

\subsubsection{Mögliche Auswirkungen des Klimawandels auf die Wasserversorgung in Hamburg}

\subsubsection{Auswirkungen des Klimawandels auf Wasserangebot, Wasserversorgungs- infrastruktur und Abwasserentsorgung}

Es liegen derzeit nur wenige Untersuchungen zu den Auswirkungen des Klimawandels auf das Wasserangebot, die Versorgungsinfrastruktur und die Abwasserentsorgung in Hamburg und der Metropolregion vor. Dennoch ist dieses Thema bereits im Aktionsplan Anpassung der Stadt Hamburg verankert. Hier wird zum einen die Problematik veränderter Niederschlagsmuster und gleichzeitig zunehmender Versiegelung angesprochen. Es wird erwartet, dass die prognostizierte Zunahme von Regenintensität und -frequenz zu zukünftigen Überlastungen der Siele und potenziellen Überflutungen sowie zu einer Zunahme der Gewässerverunreinigung durch vermehrt abfließendes Regenwasser (z. B. von Straßen) führen kann. Eine Kapazitätsanpassung der Siele soll dem entgegenwirken (Bürgerschaft der Freien und Hansestadt Hamburg 2013). In Projekten, die von der Behörde für Umwelt und Energie gefördert werden, wie z. B. RISA (RegenInfraStrukturAnpassung) und KLIQ (Klimafolgenanpassung innerstädtischer hochverdichteter Quartiere in Hamburg), sollen zudem frühzeitig geeignete Lösungsansätze entwickelt werden, damit Überflutungen und zusätzliche Gewässerbelastungen verhindert werden können. Als weiteres Ziel wird die Sicherstellung der Trinkwasserversorgung genannt. Hierfür wird eine Modellierung des zukünftig zur Verfügung stehenden Grundwassers angestrebt, sodass mögliche Knappheiten frühzeitig erkannt werden (Bürgerschaft der Freien und Hansestadt Hamburg 2013).

Eine umfangreiche Untersuchung in diesem Zusammenhang wurde im Rahmen des Forschungsprojektes GLOWA-Elbe (Globaler Wandel des Wasserkreislaufs) für das Elbeeinzugsgebiet vorgenommen. Die Ergebnisse zeigen, dass Wassermengenkonflikte infolge des Klimawandels generell entstehen können, wenn die im Rahmen des existierenden Systems von Speichern bestehenden Möglichkeiten zum Wasserrückhalt und zur gezielten Niedrigwasserauffüllung ausgeschöpft sind. Dabei ist eine zunehmende Umverteilung von Abflüssen ein Frühindikator für die Annäherung an eine solche Grenze (Wechsung et al. 2013).

Von einer klimawandelbedingten Verringerung des Wasserdargebots werden aber vermutlich nur wenige Wirtschaftssektoren und Haushalte betroffen sein. Vielmehr scheint es, dass durch die Auswirkungen eines zukünftig weiter reduzierten Wasserdargebots bereits bestehende Verknappungen verstärkt werden (Grossmann et al. 2013). Es wird allerdings erwartet, dass der geringeren Wasserverfügbarkeit im Zuge des Klimawandels mit Investitionen begegnet wird, durch die der Wasserbedarf weiter verringert und
Wasserverschmutzung reduziert wird. Möglich erscheinen diese Investitionen aufgrund der positiven wirtschaftlichen Entwicklung. Für diese Einschätzung wurden von Blazejczak et al. (2011) zwei Szenarien bis zum Jahr 2020 von den globalen IPCC-Szenarien abgeleitet und regionalisiert. Anhand eines regionalökonomischen Modells, das die Prozesse demographischer und ökonomischer Prozesse beschreibt, wird eine zukünftige Verteilung der Bevölkerung sowie von Beschäftigten und Einkommen in den Regionen des Elbeeinzugsgebietes simuliert. Diese bilden die Grundlage für die Modellierung von Landnutzung, Wassernachfrage, Abwasseraufkommen und Wasserangebot von Industrie und Energiesektor. In beiden Szenarien werden Beschäftigtenwachstumsraten von über 10 \% für die Hansestadt Hamburg simuliert, und auch in den umliegenden Regionen in der Metropolregion zeichnen sich steigende Bevölkerungszahlen ab. In ländlichen Regionen wird dagegen mit weiteren Bevölkerungs- und Beschäftigungsrückgängen gerechnet (Blazejczak et al. 2011).

Der Klimawandel führt allerdings möglicherweise dazu, dass bestehende Kapazitäten aufrechterhalten werden müssen. So wäre es möglich, dass die Dimensionierung der Wasserver- und -entsorgung trotz verringerten Wasserbedarfs nicht reduziert werden kann, weil klimawandelbedingt erwartete Extremereignisse dies erforderlich machen (Hansjürgens et al. 2013). Diese Einschätzung passt zu dem Vorhaben der Stadt Hamburg, die Kapazitäten der Siele an die erwarteten Niederschlagsänderungen anzupassen (Bürgerschaft der Freien und Hansestadt Hamburg 2013).

Für Industrien, die Elbwasser in Hamburg nutzen und von Niedrigwasser betroffen sind, werden für die Periode 2048-2051 keine Schäden erwartet - zum einen, weil industrielle Nutzer und Kraftwerke meist an Gewässerabschnitten mit einer größeren Durchflussmenge angesiedelt sind und vielfältige Anpassungsmechanismen umsetzen, zum anderen wird für diese Nutzungen in allen Szenarien ein Rückgang des Entnahmebedarfs projiziert, der im Zusammenhang mit Bevölkerungs-, Technologie- und Wirtschaftsentwicklung steht. Ökonomische Schäden werden nur für Nutzer erwartet, die das Elbwasser für die Beregnung landwirtschaftlicher Flächen nutzen, für die einstaubewässerten Feuchtgebiete sowie für die Teichwirtschaft. Die Ergebnisse zeigen, dass es sinnvoll wäre, Anpassungen im Bereich Wasserressourcenmanagement auf bereits heute in Trockenperioden auftretende Probleme in der Wasserbewirtschaftung auszurichten (Grossmann et al. 2013).

\subsubsection{Auswirkungen des Klimawandels auf die Wassernachfrage}

Für das Einzugsgebiet von HAMBURG WASSER erstellten Kluge et al. (2014) eine Wasserbedarfsprognose bis zum Jahr 2045 auf der Basis des Jahres 2011. Für die unterschiedlichen Verbrauchergruppen werden spezifische Faktoren analysiert, die Einfluss auf deren Wassernachfrage haben. So werden für Haushalte sowohl technische als auch siedlungs- und sozialstrukturelle Faktoren berücksichtigt. Für die Verbrauchergruppen Industrie, Gewerbe und Dienstleistungen werden Erwerbstätige sowie die spezifischen Wasserbedarfe einzelner Sektoren als Bezugsgröße gewählt. Entwicklungstrends werden hier anhand von Eckdaten aus gängigen Wirtschaftsprognosen herausgearbeitet. 


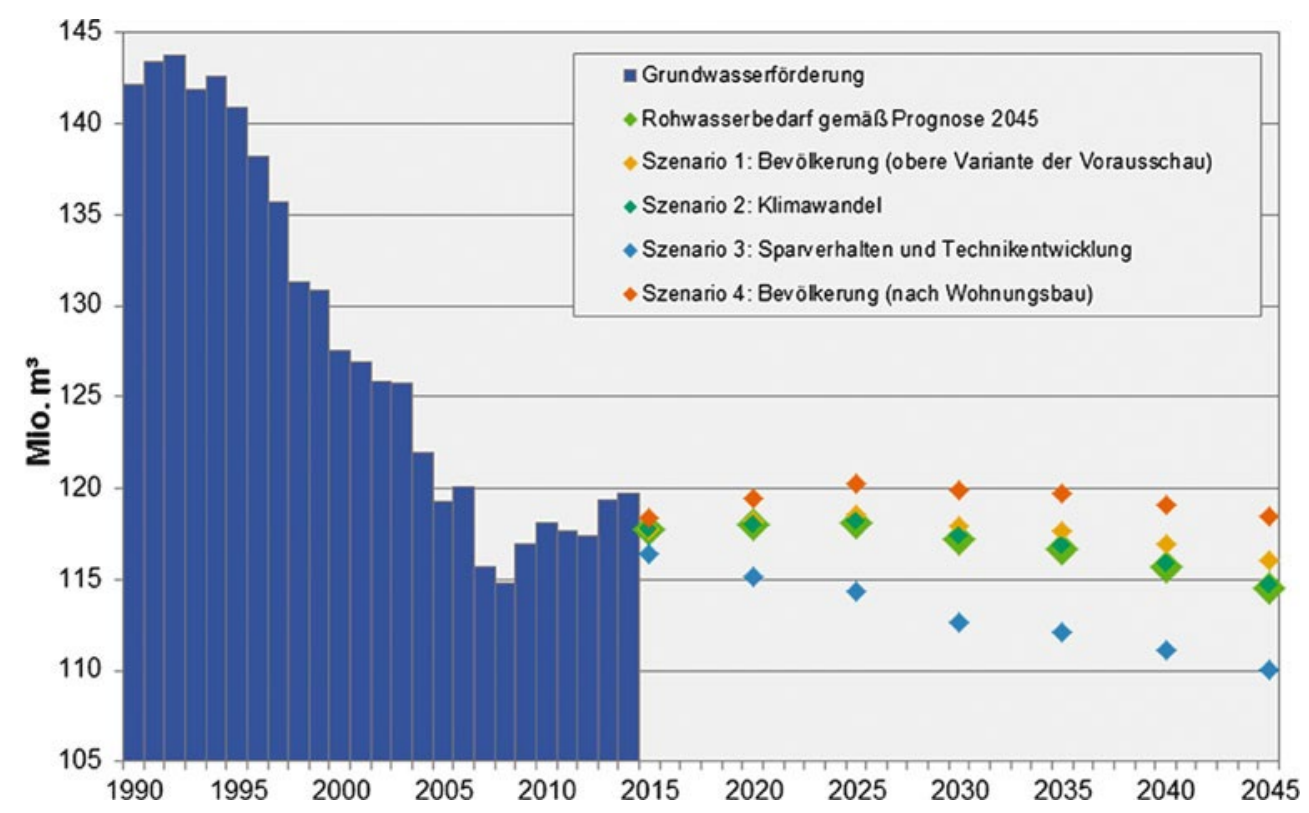

- Abb. 9.1 Szenarien zukünftiger Entwicklung des Rohwasserbedarfs in Hamburg bis 2045 in 5-JahresSchritten. (Nach Kluge et al. 2014)
Für die Wirkungsabschätzung klimatischer Veränderungen auf den Wasserbedarf werden die täglichen Wasserabgabemengen sowie kalendarische und meteorologische Einflussfaktoren in einem Modell analysiert. Im Szenario „Klimawandel“ wird von einer Zunahme längerer Phasen trockener bzw. sowohl heißer als auch trockener Tage ausgegangen. Der Gesamtwasserbedarf erhöht sich dadurch um 0,2 Mio. $\mathrm{m}^{3}$ im Vergleich zum Referenzszenario. Die Ergebnisse zeigen zudem, dass (a) die Temperaturen erst über $25^{\circ} \mathrm{C}$ exponentiell an Einfluss gewinnen, (b) der Wasserbedarf in längeren Phasen geringen Niederschlags deutlich ansteigt und (c) der Wasserbedarf in Trocken- und HitzeTrocken-Perioden deutlich ansteigt (Kluge et al. 2014). Der Einfluss des Klimawandels auf den Wasserbedarf ist allerdings relativ gering im Vergleich zu anderen Faktoren. $\bullet$ Abb. 9.1 verdeutlicht, dass die Bevölkerungsentwicklung, das Wassersparverhalten und die Technikentwicklung bis zum Jahr 2045 wesentlich bedeutender für den Rohwasserbedarf in Hamburg sein werden als die erwarteten klimatischen Veränderungen.

\subsubsection{Zwischenfazit}

Das Trinkwasser in der Stadt Hamburg wird ausschließlich aus Grundwasser gewonnen. Durch die starke Versiegelung in der Stadt wurde der natürliche Kreislauf, bei dem ein Teil des Regenwassers versickert und Grundwasser bildet, bereits gestört, da der Niederschlag vermehrt über Oberflächengewässer abfließt. Versiegelung und andere Faktoren, wie Eindeichung von Flüssen oder die Trockenlegung von Mooren, haben bereits heute vielerorts den Grundwasserspiegel sinken lassen. Trotz dieser Entwicklungen und des erwarteten Klimawandels sind für die Region Hamburg nach derzeitigem Kenntnisstand zukünftig keine Engpässe bei der öffentlichen Wasserversorgung und eventuell damit einhergehende Preiserhöhungen, wie sie beispielsweise in den USA erwartet werden, zu befürchten. Betroffen wären von einer solchen Verknappung vor allem die Haushalte, die rund $70 \%$ des Trinkwassers abnehmen. Klimawandelbedingte Schäden erscheinen dagegen eher bei Nutzern der natürlichen Wasservorräte möglich, und hier vorwiegend in der Landwirtschaft, die Elbwasser für die Beregnung nutzt und Einschränkungen durch Niedrigwasser erfahren könnte.

Änderungen durch den Klimawandel können sich zudem auf der Wassernachfrageseite ergeben. Die Wasserbedarfsprognose für Hamburg zeigt, dass durch den Klimawandel mit einer leichten Erhöhung der Wassernachfrage in Hamburg gerechnet werden muss. Dieses Ergebnis stimmt mit dem Großteil der Untersuchungen für andere deutsche Regionen überein. Ausschlaggebend hierfür sind die unter dem Klimawandel erwarteten heißeren und trockeneren Sommer sowie ein Anstieg von Hitzetagen. Für Hamburg erhöht sich der Gesamtwasserbedarf dadurch um 0,2 Mio. $\mathrm{m}^{3}$ bis 2045 im Vergleich zum Referenzszenario ohne Klimawandel.

Es wird zudem erwartet, dass klimatische Änderungen stärkere Schwankungen in der Wassernachfrage verursachen. Die Folge solcher Schwankungen können Unterauslastungen von Anlagen in Perioden mit relativ geringem Wasserbedarf sein, die zu Ineffizienzen führen. Auf der anderen Seite wird in der Literatur darauf hingewiesen, dass Kapazitäten für die Bedienung des Spitzenbedarfs aufrechterhalten werden sollten, wozu beispielsweise auch das Vorhalten von Löschwasser zählt. Die Trinkwasserbehälter dienen bereits heute als Puffer für die ständig schwankende Wasserentnahme. Sie leeren sich bei hohem Bedarf (tagsüber) und füllen sich bei geringem Bedarf (nachts).

Verschiedene Studien zeigen für Regionen in Deutschland und auch für Hamburg allerdings, dass zukünftig andere Faktoren wie die allgemeine Bevölkerungsentwicklung und technologische Veränderungen einen wesentlich größeren Einfluss auf die Wassernachfrage haben werden als der Klimawandel.

Herausforderungen durch den Klimawandel können bei der Ableitung von Regenwasser insbesondere bei Starkregenereignissen auftreten, die im Zuge des Klimawandels den Projektionen zufolge auch im Hamburger Raum vermehrt auftreten werden. Auch wenn zu den Auswirkungen auf die Wasserinfrastruktur keine direkten wissenschaftlichen Untersuchungen für Hamburg vorliegen, ist die Stadt bereits dabei, die Kapazitäten der Siele anzupassen. Durch eine Überlastung der Siele können Über- 
schwemmungen auftreten. Diese können zu Schäden an Gebäuden, Infrastrukturen und anderen Vermögensgegenständen führen. Darüber hinaus werden insbesondere durch das Überlaufen der Mischsiele - ein Viertel der Siele in Hamburg führen Regen- und Schmutzwasser gemeinsam - Schadstoffe über den Oberflächenablauf transportiert, die zu Verunreinigungen in Gewässern führen können.

Eine Untersuchung des Augusthochwassers 2002 in Sachsen und Sachsen-Anhalt hat gezeigt, dass durch starke Überschwemmungsereignisse erhebliche Schäden an der Wasserversorgungsinfrastruktur entstehen können, durch die auch die Wasserqualität beeinträchtigt werden kann. Die Beeinträchtigung der Wasserversorgung wurde dabei durch Schäden an Anlagen der Energie-, Mess-, Steuer- und Regelungstechnik, an Brunnen- und Fassungsanlagen, an Wasseraufbereitungsanlagen sowie am Rohrnetz verursacht. Da große Teile Hamburgs in sturmflut- oder binnenhochwassergefährdeten Gebieten liegen, wäre ein Ereignis mit ähnlichen Folgen auch für Hamburg denkbar. Vorkehrungen für einen Notfall, bei dem ein Wasserwerk ausfällt, sind seitens des Hamburger Wasserversorgers bereits getroffen worden, indem sich das Wasser innerhalb des Verbunds umleiten lässt, da jede größere Verbrauchszone von mehreren Wasserwerken beliefert wird.

Die bisherigen Untersuchungen für Hamburg geben bereits einen Einblick in die Herausforderungen, die sich der Wasserwirtschaft durch den Klimawandel stellen. Vor allem die Entwicklung der Wassernachfrage, die auch durch den Klimawandel beeinflusst wird, wurde detailliert für Hamburg untersucht. Allerdings zeigt sich, dass noch weiterer Forschungsbedarf besteht. Bisher liegen keine flächendeckenden Analysen für die Auswirkungen des Klimawandels auf das Wasserangebot und die Wasserversorgungsinfrastrukturen vor - weder für Hamburg noch für die Metropolregion Hamburg. Darüber hinaus fehlen monetäre Bewertungen für Schadensereignisse wie Überschwemmungen und damit verbundene Überlastungen der Siele oder Ausfälle der Wasserversorgung. Diese wären für die Bewertung von Anpassungsmaßnahmen beispielsweise anhand von Kosten-Nutzen-Analysen notwendig. Zudem gibt es keine wissenschaftlichen Erkenntnisse zur zukünftigen Ausgestaltung von Infrastrukturanlagen, die auch auf die Herausforderungen des Klimawandels ausgerichtet sind. Es gibt allerdings Forschungsinitiativen, die hier in naher $\mathrm{Zu}$ kunft Erkenntnisse liefern können, wie beispielsweise Projekte der Forschungsinitiative INIS (Intelligente und multifunktionelle Infrastruktursysteme für eine zukunftsfähige Wasserversorgung und Abwasserentsorgung) des Bundesministeriums für Bildung und Forschung (Deutsches Institut für Urbanistik gGbmH 2015). Auch die Arbeit des Department Urban Water Management der schweizerischen Forschungseinrichtung EAWAG könnte übertragbare Ergebnisse liefern (u. a. Veronesi et al. 2013).

\section{$9.4 \quad$ Fazit}

Es zeigt sich zusammenfassend für Hamburg und die Metropolregion, dass es ratsam ist, Produktions- und Versorgungsinfrastrukturen mittel- bis langfristig an die Folgen des Klimawandels anzupassen. Da eine Umsteuerung in diesen Infrastrukturen nur in längerfristigen Zeitmaßstäben zu verwirklichen ist, wird deutlich, wie wichtig es ist, bereits heute Abschätzungen zu den Auswirkungen des Klimawandels vorzunehmen und ggf. entsprechende Anpassungen zu planen.

Basierend auf der Auswertung des aktuellen Wissensstandes zu klimawandelbedingten Betroffenheiten des Energiesektors zeigt sich, dass die meisten Bereiche der Wertschöpfungskette negativ betroffen sein werden. Als wesentliche Einflussfaktoren konnten die Wasserverfügbarkeit, Extremwetterereignisse und steigende Durchschnittstemperaturen identifiziert werden. Auch wenn sich bei einzelnen Aspekten unterschiedliche Einschätzungen gezeigt haben, so kann doch festgehalten werden, dass die Betroffenheit der Energieversorgung insgesamt als grundsätzlich gut handhabbar anzusehen ist. Zudem ist deutlich geworden, dass die Netzinfrastruktur die verletzlichste Komponente des Energiesystems ist. Somit ist zu erwarten, dass im Energiesektor insbesondere Investitionen in die Anpassung der Anlagen sowie der Übertragungs- und Verteilnetze notwendig sind.

Aus den bisherigen Studien geht zudem hervor, dass der Wassersektor in Hamburg vor allem im Bereich der Abwasserentsorgung durch eine Zunahme von Starkregenereignissen und im Bereich der Wassernachfrage durch einen Anstieg von Hitzeperioden betroffen ist. Daraus ergibt sich, dass bei der Anpassung an den Klimawandel besonderes Augenmerk auf die Sielkapazitäten für die Ableitungen von Regenwasser bei Starkregenereignissen gelegt werden sollte. Darüber hinaus zeigt sich mit Blick auf den Klimawandel die Relevanz einer Überprüfung der Wasserversorgungsinfrastruktur auf ihre Hochwassersicherheit in den entsprechend gefährdeten Gebieten sowie auf die steigenden Anforderungen durch stärkere Schwankungen in der Wassernachfrage.

Im Hinblick auf spezifische Aussagen zu Betroffenheiten der Energie- und Wasserversorgung in Hamburg und der Metropolregion bestehen noch Wissenslücken und Forschungsbedarf, da hierzu bislang kaum wissenschaftliche Untersuchungen durchgeführt wurden. Des Weiteren hat sich gezeigt, dass bislang auch noch ein umfassender Forschungsbedarf zu monetären Abschätzungen von klimawandelbedingten Betroffenheiten bei Versorgungsinfrastrukturen besteht. Dies gilt auch für die notwendige Berücksichtigung von Klimawandelfolgen im Rahmen der zukünftigen Ausgestaltung regulatorischer Rahmenbedingungen und damit verbundener ökonomischer Anreizsetzungen.

\section{Literatur}

\section{Energieversorgung}

Bardt $\mathrm{H}$, Biebeler H, Haas H (2013) Einfluss des Klimawandels auf die deutsche Energieversorgung. Wirtschaftsdienst 93(5):307-315

Barstad I, Sorteberg A, dos-Santos Mesquita M (2012) Present and future offshore wind power potential in northern Europe based on downscaled global climate runs with adjusted SST and sea ice cover. Renew Energ 44:398-405

Birkmann J, Bach C, Guhl S, Witting M, Welle T, Schmude M (2010) State of the Art der Forschung zur Verwundbarkeit kritischer Infrastrukturen am Beispiel Strom/Stromausfall. Freie Universität Berlin, Berlin (Webseiten der Freien Universität Berlin)

Bothe D, Riechmann C (2008) Hohe Versorgungszuverlässigkeit bei Strom wertvoller Standortfaktor für Deutschland. Energiewirtsch Tagesfr 58(10):31-36 
Bundesministerium des Innern (2009) Nationale Strategie zum Schutz Kritischer Infrastrukturen (KRITIS-Strategie). BMI, Berlin (Webseiten des Bundesministeriums des Innern)

Bundesministerium für Verkehr, Bau und Stadtentwicklung (2007) Schifffahrt und Wasserstraßen in Deutschland - Zukunft gestalten im Zeichen des Klimawandels: Bestandsaufnahme. BMVI, Bonn (Webseiten des Bundesministeriums für Verkehr, Bau und Stadtentwicklung)

Bundesnetzagentur (2006) Untersuchungsbericht über die Versorgungsstörungen im Netzgebiet des RWE im Münsterland vom 25.11.2005. Forschungsbericht. Bundesnetzagentur, Bonn (Webseiten der Bundesnetzagentur)

Bundesnetzagentur (2014) Monitoringbericht 2014. Bundesnetzagentur, Bonn (Webseiten der Bundesnetzagentur)

Bundesregierung (2008) Deutsche Anpassungsstrategie an den Klimawandel. Bundesregierung, Bonn (Webseiten der Bundesregierung)

Bürgerschaft der Freien und Hansestadt Hamburg (2013a) Aktionsplan Anpassung an den Klimawandel. Mitteilung des Senats an die Bürgerschaft. Drucksache 20/8492. Bürgerschaft der Freien und Hansestadt Hamburg, Hamburg (Webseiten der Bürgerschaft der Freien und Hansestadt Hamburg)

Bürgerschaft der Freien und Hansestadt Hamburg (2013b) Masterplan Klimaschutz - Zielsetzung, Inhalt und Umsetzung. Mitteilung des Senats an die Bürgerschaft. Drucksache 20/8493. Bürgerschaft der Freien und Hansestadt Hamburg, Hamburg (Webseiten der Bürgerschaft der Freien und Hansestadt Hamburg)

Buth M, Kahlenborn W, Savelsberg J, Becker N, Greiving S, Fleischhauer M, Zebisch M, Schneiderbauer S, Schauser I (2015) Vorbereitungspapier zur Fachkonferenz Vulnerabilität Deutschlands gegenüber dem Klimawandel des Netzwerks Vulnerabilität am 1. Juni 2015 in Berlin. Sektorenübergreifende Analyse des Netzwerks Vulnerabilität

CDP Worldwide (2014a) Cities program (Webseiten des CDP)

CDP Worldwide (2014b) CDP Cities 2014 Information Request (Webseiten des (DP)

CDP Worldwide (2014c) CDP Cities 2014 Information Request Hamburg (Webseiten des CDP)

Cortekar J, Groth M (2013) Der deutsche Energiesektor und seine mögliche Betroffenheit durch den Klimawandel - Synthese der bisherigen Aktivitäten und Erkenntnisse. Climate Service Center, Hamburg

Cortekar J, Groth M (2015) Adapting energy infrastructure to climate change - Is there a need for government interventions and legal obligations within the German "Energiewende"? Energy Procedia 73:12-17

Deb AK (2000) Powerline Ampacity system theory, modeling, and applications. CRC Press, Boca Raton

Deutscher Bundestag (2011) Gefährdung und Verletzbarkeit moderner Gesellschaften am Beispiel eines großräumigen und langandauernden Ausfalls der Stromversorgung. Bericht des Ausschusses für Bildung, Forschung und Technikfolgenabschätzung (Webseiten des Deutschen Bundestags)

Dunkelberg E, Hirschl B, Hoffman E (2009) Ergebnis des Stakeholderdialogs zu Chancen und Risiken des Klimawandels - Energiewirtschaft

Energieportal Hamburg (2015) Hamburg in Zahlen (Webseiten des Energieportals Hamburg)

Engelhard P (2011) Erwartungen an die Anpassungspolitik. Vortrag auf dem Workshop der Forschungsgruppe Chamäleon und dem Bundesverband der Energie- und Wasserwirtschaft (BDEW), Berlin. http://www. climate-chameleon.de/htm/documents/DokumentationEnergieWorkshop_11-06-27_final.pdf. Zuletzt zugegriffen am 27.06.2011, 05.4.2011.

Eskeland G, Jochem E, Neufeldt H, Traber T, Rive N, Behrens A (2008) The future of European electricity - choices before 2020. CEPS policy briefs

Europäische Kommission (2007) Grünbuch der Kommission an den Rat, das Europäische Parlament, den Europäischen Wirtschafts- und Sozialausschuss und den Ausschuss der Regionen - Anpassung an den Klimawandel in Europa - Optionen und Maßnahmen der EU. Europäische Kommission, Brüssel (Webseiten der Europäischen Kommission)

Finley T, Schuchard R (2009) Adapting to climate change - a guide for the energy and utility industry (Webseiten der BSR)

Förster H, Lilliestam J (2010) Modeling thermoelectric power generation in view of climate change. Reg Environ Change 10:327-338

Groth M, Cortekar J (2014) Climate change adaptation strategies within the framework of the German "Energiewende" - Is there a need for government interventions and legal obligations? University of Lüneburg Working Paper Series in Economics. Working Paper 315. University of Lüneburg, Lüneburg Groth M, Cortekar J (2015) Die Relevanz von Klimawandelfolgen für Kritische Infrastrukturen am Beispiel des deutschen Energiesektors. University of Lüneburg Working Paper Series in Economics. Working Paper 335. University of Lüneburg, Lüneburg

Growitsch C, Malischek R, Nick S, Wetzel H (2015) The costs of power interruptions in Germany: a regional and sectoral analysis. Ger Econ Rev 16(3):307-323

Hirschl B, Dunkelberg E (2009) Problemaufriss - Auswirkungen des Klimawandels auf die Energiewirtschaft. Vortrag bei dem Stakeholderdialog zu Chancen und Risiken des Klimawandels - Energiewirtschaft, Dessau, 30.6.2009.

Hoffmann B, Häfele S, Karl U (2012) Analysis of performance losses of thermal power plants in Germany - A system dynamics model approach using data from regional climate modelling. Energy 49:193-203

Hu PT, Negnevitsky M, Kashem MA (2006) Loading capabilities assessment of power transmission lines. Australasian Universities Power Engineering Conference (AUPE'06), Melbourne, S 1-5

IMPACT2C (2016) Solar photovoltaics potential (Webseiten des Projektes IMPACT2C)

Jayant S, Dale L, Fitts G, Larsen P, Koy K, Lewis S, Lucena A (2011) Estimating risk to California energy infrastructure from projected climate change. California Energy Commission, Sacramento (Publication number: CEC500-2011-XXX)

Koch H, Vögele S (2013) Hydro-climatic conditions and thermoelectric electricity generation - Part I: development of models. Energy 63:42-51

Koch H, Vögele S, Hattermann F, Huang S (2014) Hydro-climatic conditions and thermoelectric electricity generation - Part II: model application to 17 nuclear power plants in Germany. Energy 69:700-707

Koch H, Vögele S, Hattermann F, Huang S (2015) The impact of climate change and variability on the generation of electrical power. Meteorol $Z$ 24(N2.2):173-188

Kuckshinrichs W, Fischedick M, Venjakob J, Fichtner W, Rothstein B (2008) Annex 2. In: Deutsche Anpassungsstrategie (DAS) an den Klimawandel - Bericht zum Nationalen Symposium zur Identifizierung des Forschungsbedarfs

Lauwe P (2009) Die kritische Infrastruktur Energieversorgung. Vortrag bei dem Stakeholderdialog zu Chancen und Risiken des Klimawandels - Energiewirtschaft, Dessau, 30.6.2009

Linnerud K, Mideksa T, Eskeland G (2011) The impact of climate change on nuclear power supply. Energ J 32:149-168

Piaszeck S, Wenzel L, Wolf A (2013) Regional diversity in the costs of electricity outages - results for German counties. In: HWWI Research Paper 142. Hamburgisches WeltWirtschaftsInstitut (HWWI), Hamburg

Polkehn-Appel A (2015) Auskunft per Email der Pressesprecherin der Stromnetz Hamburg GmbH auf eine Anfrage zur Möglichkeit der Verwendung des Netzzustandsberichts 2014 und des Strukturberichts 2014 vom 16. Juli 2015

Pryor SC, Barthelmie RJ, Kjellström E (2005a) Potential climate change impact on wind energy resources in northern Europe: analyses using a regional climate model. Clim Dynam 25:815-835

Pryor SC, Schoof JT, Barthelmie RJ (2005b) Climate change impacts on wind speeds and wind energy density in northern Europe: empirical downscaling of multiple AOGCMs. Clim Res 29:183-198

Rothstein B, Halbig G (2010) Weather sensitivity of electricity supply and data services of the German met office. Manag Weather Clim Risk Energy Ind. S 253-265

Rubbelke D, Vogele S (2011) Impacts of climate change on european critical infrastructures: the case of the power sector. Environ Sci Policy 14:53-56

Schaeffer R, Szklo AS, de Lucena AFP, Borba BSMC, Nogueira LPP, Fleming FP, Troccoli A, Harrison M, Boulahya MS (2012) Energy sector vulnerability to climate change: a review. Energy, Bd. 38., S 1-12

Scheele U, Oberdörffer J (2011) Transformation der Energiewirtschaft - Zur Raumrelevanz von Klimaschutz und Klimaanpassung

Schmitt R (2012) Schneelast an Stromleitungen - Heute und in Zukunft. Climate Service Center, Hamburg

Statistisches Amt für Hamburg und Schleswig-Holstein (2014) Stromerzeugung in Hamburg 2013 (Webseiten des Statistischen Amts für Hamburg und Schleswig-Holstein) 
Stephen R, Douglas D, Gaudry M (2002) Thermal behavior of overhead conductors. CIGRE Report 207. Cigré, Paris

Stromnetz Hamburg GmbH (2014) Technische Daten. Stromverteilnetz Hamburg. http://daten.transparenz.hamburg.de/Dataport.HmbTG.ZS.Webservice.GetRessource100/GetRessource100.svc/e1f7a240-784c-4eeb83f8-1f889d88a06a/Upload_Technische_Daten_Stromnetz_Hamburg. pdf. Zuletzt zugegriffen am 30.10.2015

Stromnetz Hamburg GmbH (2015) Wie der Strom zu den Hamburgern kommt (Webseiten der Stromnetz Hamburg GmbH)

US-Canada Power System Outage Task Force (Hrsg) (2004) Final Report on the August 142003 Blackout in the United States and Canada - Causes and Recommendations, Washington, Ottawa

Vine E (2012) Adaptation of California's electricity sector to climate change. Clim Change 111:75-99

van Vliet MTH, Yearsley JR, Ludwig F, Vögele S, Lettenmaier DP, Kabat P (2012) Vulnerability of US and European electricity supply to climate change. Nat Clim Chang 2:676-681

Wachsmuth J, von Gleich A, Gößling-Reisemann S, Lutz-Kunisch B, Stührmann S, Gabriel J, Meyer S (2012) Kapitel 4.8 - Energiewirtschaft. In: Schuchardt B, Wittig S (Hrsg) Vulnerabilität der Metropolregion Bremen-Oldenburg gegenüber dem Klimawandel (Synthesebericht). Nordwest2050-Berichte 2. Projektkonsortium ,nordwest2050'., Bremen, Oldenburg, S 94-111

Wachsmuth J, Blohm A, Gößling-Reisemann S, Eickemeier T, Ruth M, Gasper R, Stührmann S (2013) How will renewable power generation be affected by climate change? The case of a metropolitan region in northwest Germany. Energy 58:192-201

\section{Wasserversorgung}

Ansmann T (2013) Szenarien zur Wassernachfrage der öffentlichen Wasserversorgung. In: Wechsung F, Hartje V, Kaden S, Venohr M, Hansjürgens B, Gräfe P (Hrsg) Die Elbe im globalen Wandel - Eine integrative Betrachtung. Konzepte für eine nachhaltige Entwicklung einer Flusslandschaft, Bd. 9. Weissensee Verlag, Berlin, S 319-340

Blazejczak J, Gornig M, Hartje V (2011) Downscaling nonclimatic drivers for surface water vulnerabilities in the Elbe river basin. Reg Environ Change 12(1):69-80

Braubach A (2011) Vulnerabilität der kritischen Infrastruktur Wasserversorgung gegenüber Naturkatastrophen - Auswirkungen des Augusthochwassers 2002 auf die Wasserversorgung und das Infektionsgeschehen der Bevölkerung in Sachsen und Sachsen-Anhalt. Bundesamt für Bevölkerungsschutz und Katastrophenhilfe: Forschung im Bevölkerungsschutz, Bd. 12. (Webseiten des Bundesamts für Bevölkerungsschutz und Katastrophenhilfe)

Bürgerschaft der Freien und Hansestadt Hamburg (2013) Aktionsplan Anpassung an den Klimawandel. Mitteilung des Senats an die Bürgerschaft. Drucksache 20/8492. Bürgerschaft der Freien und Hansestadt Hamburg, Hamburg (Webseiten der Stadt Hamburg)

Dannenberg A, Mennel T, Osberhaus D, Sturm B (2009) The economics of adaptation to climate change - the case of Germany. Diskussionspapier 09-057. Zentrum für Europäische Wirtschaftsforschung (ZEW), Mannheim (Webseiten des ZEW)

Der Beauftragte der Bundesregierung für die neuen Bundesländer (2011) Daseinsvorsorge im demografischen Wandel zukunftsfähig gestalten - Handlungskonzept zur Sicherung der privaten und öffentlichen Infrastruktur in vom demografischen Wandel besonders betroffenen ländlichen Räumen. Der Beauftragte der Bundesregierung für die neuen Bundesländer, Berlin (Webseiten der Beauftragten der Bundesregierung für die neuen Bundesländer)

Deutsches Institut für Urbanistik gGmbH (2015) Intelligente und multifunktionelle Infrastruktursysteme für eine zukunftsfähige Wasserversorgung und Abwasserentsorgung - Zwischenergebnisse aus den INIS-Projekten. difu, Berlin (Webseiten des difu)

Downing TE, Butterfield RE, Edmonds B, Knox JW, Moss S, Piper BS, Weatherhead EK, CCDeW Projektteam (2003) Climate change and demand for water. Research report. Stockholm Environment Institute Oxford Office, Stockholm

EEA (2007) Climate change and water adaptation issues. EEA-Report 2/2007. European Environment Agency, Kopenhagen (Webseiten der EEA)
Grossmann M, Koch H, Lienhoop N, Vögele S, Mutafoğlu K, Dietrich O, Möhring J, Kaltofen M (2013) Ökonomische Bewertung von Wasserdefiziten: ein integrierter ökonomisch-hydrologischer Modellansatz. In: Wechsung $F$, Hartje V, Kaden S, Venohr M, Hansjürgens B, Gräfe P (Hrsg) Die Elbe im globalen Wandel - Eine integrative Betrachtung. Konzepte für eine nachhaltige Entwicklung einer Flusslandschaft, Bd. 9. Weissensee Verlag, Berlin, S 443-477

HAMBURG WASSER (2015a) Unser Wasser - Trinkwasser und Abwasser in der Hansestadt Hamburg. HAMBURG WASSER, Hamburg (Webseiten von HAMBURG WASSER)

HAMBURG WASSER (2015b) Zahlen zur Wasserversorgung: Versorgungsnetz in Zahlen. HAMBURG WASSER, Hamburg. http://www.hamburgwasser. de/wasserversorgung-in-zahlen.html. Zuletzt zugegriffen am 30.10.2015

HAMBURG WASSER (2015c) Die Wasserwerke im Bereich Mitte/Ost - Geschichte, Wasserförderung, Aufbereitung. HAMBURG WASSER, Hamburg (Webseiten von HAMBURG WASSER)

HAMBURG WASSER (2015d) Abwasserentsorgung in Zahlen. HAMBURG WASSER, Hamburg. http://www.hamburgwasser.de/abwasserentsorgung-inzahlen.html. Zuletzt zugegriffen am 30.10.2015

Hanemann WM, Dale L (2006) Economic damages from climate change: an assessment of market impacts. Working Paper 1029. Department of Agricultural and Resource Economics UCB, Berkeley

Hansjürgens B, Wechsung F, Gräfe P (2013) Szenarien und Wirkungsanalyse - eine integrative Schlussbetrachtung für das Elbeeinzugsgebiet. In: Wechsung F, Hartje V, Kaden S, Venohr M, Hansjürgens B, Gräfe P (Hrsg) Die Elbe im globalen Wandel - Eine integrative Betrachtung, Konzepte für eine nachhaltige Entwicklung einer Flusslandschaft, Bd. 9. Weissensee Verlag, Berlin, S 591-596

Kersting M, Werbeck N (2013) Trinkwasser und Abwasser in Zeiten des Wandels - Eine Szenarienbetrachtung für die dynaklim-Region. dynaklim-Publikation Nr. 39. (Webseiten des dynaklim-Projekts)

Kluge T, Liehr S, Schulz O, Sunderer G, Wackerbauer J, Lippelt J (2014) Wasserbedarfsprognose 2045 für das Versorgungsgebiet von HAMBURG WASSER -Aktualisierung der Wasserbedarfsprognose 2030 für das Versorgungsgebiet der Hamburger Wasserwerke GmbH (HWW) in der Metropolregion Hamburg. Institut für sozial-ökologische Forschung (ISOE) GmbH, Frankfurt am Main, München

Koch H, Grünewald U (2011) Anpassungsoptionen der Wasserbewirtschaftung an den globalen Wandel in Deutschland. acatech-Materialien Nr. 5. acatech, München (Webseiten von acatech)

Mikat H, Wagner H, Roth U (2010) Wasserbedarfsprognose für Südhessen 2100 Langfristige Prognose im Rahmen eines Klimafolgen-Projektes. gwf Wasser Abwasser 151(12):1178-1186 (Webseiten der Hessenwasser GmbH und Co. KG)

Roth U, Mikat H, Wagner H (2011) Prognose zur Entwicklung des Spitzenwasserbedarfs unter dem Einfluss des Klimawandels - Eine Abschätzung am Beispiel der hessischen Landeshauptstadt Wiesbaden. gwf Wasser Abwasser 152(1):94-100

Scherzer J, Grigoryan G, Schultze B, Stadelbacher V, Niederberger J, Pöhler H, Disse M, Jacoby C, Heinisch T (2010) WASKlim - Entwicklung eines übertragbaren Konzeptes zur Bestimmung der Anpassungsfähigkeit sensibler Sektoren an den Klimawandel am Beispiel der Wasserwirtschaft. Texte 47/2010. Umweltbundesamtes, Dessau-Roßlau (Webseiten des Umweltbundesamts)

Semadeni-Davies A, Hernebring C, Svensson G, Gustafsson LG (2008) The impacts of climate change and urbanisation on drainage in Helsingborg, Sweden: combined sewer system. J Hydrol 350:100-113

Statistisches Bundesamt (2014) Umweltnutzung und Wirtschaft - Tabellen zu den Umweltökonomischen Gesamtrechnungen Teil 4: Rohstoffe, Wassereinsatz, Abwasser, Abfall. Statistisches Bundesamt, Wiesbaden (Webseiten des Statistischen Bundesamts)

Titus JG (1992) The cost of climate change to the United States. Ursprünglich erschienen. In: Majumdar SK, Kalkstein LS, Yarnal B, Miller EW, Rosenfeld LM (Hrsg) Global climate change: implications, challenges, and mitigation measures. Academy of Sciences, Pennsylvania, S 385-409

Tränckner J, Koegst T, Nowack M (2012) Auswirkungen des demographischen Wandels auf die Siedlungsentwässerung (DEMOWAS). Abschlussbericht. 
Technische Universität Dresden, Dresden (Webseiten des DEMOWASProjekts)

Veronesi M, Chawla F, Maurer M, Lienert J (2013) Climate change and the willingness to pay to reduce ecological and health risk from wastewater flooding in urban centers and the environment. Working Paper Series 1. Department of Economics, University of Verona, Verona

Wechsung F, Hansjürgens B, Hartje V, Kaden S, Venohr M (2013) Klimaverletzlichkeit des Flussgebiets Elbe. In: Wechsung F, Hartje V, Kaden S, Venohr M, Hansjürgens B, Gräfe P (Hrsg) Die Elbe im globalen Wandel - Eine integrative Betrachtung, Konzepte für eine nachhaltige Entwicklung einer Flusslandschaft, Bd. 9. Weissensee Verlag, Berlin, S 1-31

Wricke B, Tränckner J, Böhler E (2003) Dokumentation von typischen Schäden und Beeinträchtigungen der Wasserversorgung durch Hochwasserereignisse, Ableitung von Handlungsempfehlungen. Studie. DVGW, Sächsisches Ministerium für Umwelt und Landwirtschaft, Dresden (Webseiten des DVGW)

Open Access Dieses Kapitel wird unter der Creative Commons Namensnennung - Nicht kommerziell 4.0 International Lizenz (http://creativecommons.org/licenses/by-nc/4.0/deed.de) veröffentlicht, welche die nicht-kommerzielle Nutzung, Vervielfältigung, Bearbeitung, Verbreitung und Wiedergabe in jeglichem Medium und Format erlaubt, sofern Sie den/die ursprünglichen Autor(en) und die Quelle ordnungsgemäß nennen, einen Link zur Creative Commons Lizenz beifügen und angeben, ob Änderungen vorgenommen wurden.

Die in diesem Kapitel enthaltenen Bilder und sonstiges Drittmaterial unterliegen ebenfalls der genannten Creative Commons Lizenz, sofern sich aus der Abbildungslegende nichts anderes ergibt. Sofern das betreffende Material nicht unter der genannten Creative Commons Lizenz steht und die betreffende Handlung nicht nach gesetzlichen Vorschriften erlaubt ist, ist auch für die oben aufgeführten nicht-kommerziellen Weiterverwendungen des Materials die Einwilligung des jeweiligen Rechteinhabers einzuholen. 